\title{
Non-classical polar unitals in finite Dickson semifield planes
}

\author{
Alice M. W. Hui, Hiu Fai Law, Yee Ka Tai \\ and Philip P. W. Wong
}

\begin{abstract}
We give three proofs, two intrinsic and one extrinsic, that every Dickson-Ganley unital $\mathcal{U}(\sigma)$, parametrized by a field automorphism $\sigma$, is non-classical if $\sigma$ is not the identity, extending a result of Ganley's (Math Z 128:34-42, 1972); we prove that $\mathcal{U}\left(\sigma_{1}\right)$ is isomorphic to $\mathcal{U}\left(\sigma_{2}\right)$ if and only if $\sigma_{1}=\sigma_{2}$ or $\sigma_{1}=\sigma_{2}^{-1}$; and we determine the (design) automorphism group of $\mathcal{U}(\sigma)$ as the collineation subgroup of the ambient Dickson semifield plane stabilizing the unital. This contains as a special case the corresponding result of O'Nan's (J Algebra 20:495-511, 1965) on the classical unital.
\end{abstract}

Mathematics Subject Classification (2000). 51A35, 05B05, 17A35, 51A10, $51 \mathrm{~A} 45$.

Keywords. Dickson semifield plane, Dickson-Ganley polar unital, O'Nan configuration, Wilbrink's conditions, automorphism group, extension and isomorphism theorems.

\section{Introduction}

A unital $U$ is a $t-(v, k, \lambda)$ design, where $t=2, v=m^{3}+1, k=m+1$, and $\lambda=1$ : there is exactly one block on two points, there are $m+1$ points on each block, and there is a total of $m^{3}+1$ points. Let $\Pi$ be a projective plane, i.e. a $2-\left(n^{2}+n+1, n+1,1\right)$ design. A unitary polarity $\rho$ of $\Pi$ is an involutory correlation with $(\sqrt{n})^{3}+1$ absolute points. The absolute points and non-absolute lines of $\rho$ constitute an example of $U$. In this case $U$ is called a polar unital. If $\Pi$ is the classical plane $P G\left(2, q^{2}\right)$ coordinatized by the finite field $\mathbb{F}_{q^{2}}$, then $U$ is called a classical unital of order $q$. As a subvariety of $P G\left(2, q^{2}\right)$ a classical unital is a hermitian curve $[7,15,17]$.

This work was partially supported by a grant from the Research Grant Council of the HKSAR, China (Project number: HKU7060/11P). 
The problem of characterizing polar unitals seems difficult, as it involves the questions of embedding as well as the existence of unitary polarities in a projective plane. As a starting point one considers the characterization of the classical unital. A fundamental result is the determination of the automorphism group of a classical unital as a design. In [22], this is shown to be $P \Gamma U\left(3, q^{2}\right)$. In the same paper, it is also shown that in the design there is never a configuration of four blocks in general position intersecting in six points, which is now referred to as an O'Nan configuration. In [25], it is conjectured that if a unital does not admit any O'Nan configurations then it is classical. The conjecture remains open. A weaker yet remarkable result in this direction is Wilbrink's characterization of a classical unital [30]. There are three characterization conditions. In this paper we shall be concerned with the first two. The first condition is the absence of an O'Nan configuration, and the second condition is a notion of parallelism in unitals which will be recalled later in the paper. It is shown in [30] that if the order of the unital is even, then these two conditions imply the third.

Since all unitary polarities in $P G\left(2, q^{2}\right)$ are projectively equivalent, a polar unital in the classical plane is the classical unital. Consider now a polar unital $U$ embedded in a non-classical plane $\Pi$. As remarked in [20], the question as to whether $U$ can be classical is not yet answered. It is shown in that paper that a class of polar unitals embedded in the finite Figueroa planes are not classical. Here we turn our attention to a class of polar unitals embedded in the Dickson semifield planes. In [12], Ganley showed that the projective plane $\Pi(\mathcal{K})$ defined over a Dickson semifield $\mathcal{K}$ admits a unitary polarity which thus defines a polar unital $\mathcal{U}$. The unital $\mathcal{U}=\mathcal{U}(\sigma)$ is parametrized by a non-identity field automorphism $\sigma$. In [13] (see also [1]) Ganley studied the collineation subgroup $\operatorname{Col}(\mathcal{U})$ of $\Pi(\mathcal{K})$ stabilizing $\mathcal{U}$, and proved that in some special cases these polar unitals are not classical by demonstrating the existence of O'Nan configurations.

In this paper, we begin by proving the existence of O'Nan configurations in all cases. We also provide an alternative proof by demonstrating the invalidity of the second condition of Wilbrink's in these unitals.

Next we note that a Dickson-Ganley unital meets the line at infinity at one point, $(\infty)$, and $\operatorname{Col}(\mathcal{U})$ acts transitively on the affine points of the unital [13]. Such a unital is also called a transitive parabolic unital (see for example $[1])$. For our purpose the point $(\infty)$ is special for a further reason. It satisfies Wilbrink's second condition (called condition (II) in [30]) in strong form (see Corollary 4.2 below). This makes it possible to construct from $\mathcal{U}$ a design $\mathcal{S}$ which is isomorphic to the residual of a classical inversive plane.

The classical circle geometry allows us to work out the algebra to show that an isomorphism between two Dickson-Ganley polar unitals determines an isotopism between their corresponding Dickson semifields. Since two semifields define isomorphic projective planes if and only if the semifields are isotopic, this has the consequence that $\mathcal{U}\left(\sigma_{1}\right)$ is isomorphic to $\mathcal{U}\left(\sigma_{2}\right)$ if and only if $\sigma_{1}=\sigma_{2}$ or 
$\sigma_{1}=\sigma_{2}^{-1}[2,6,26]$. As our method of proof extends to the case of the identity automorphism, this provides a third and extrinsic proof that $\mathcal{U}(\sigma)$ is not classical.

Finally, we prove that the isotopism above gives rise to an isomorphism between the ambient Dickson semifield planes. As a consequence we conclude that the automorphism group $\operatorname{Aut}(\mathcal{U})$ of the design $\mathcal{U}$ equals $\operatorname{Col}(\mathcal{U})$. When $\sigma$ is the identity automorphism, this gives O'Nan's result [22] for the classical unital.

In the next section, we recall the construction of the Dickson semifield plane and the Dickson-Ganley unital as well as their basic properties. In Sect. 3 we extend Ganley's result and show that O'Nan configurations exist in all Dickson-Ganley unitals, with the consequence that these unitals are not classical. In Sect. 4 we prove that Wilbrink's condition (II) does not hold at any affine absolute point, thus giving an alternative proof of the same conclusion. We also prove that Wilbrink's condition (II) holds at the absolute point $(\infty)$ in a strong form. In Sect. 5 we recall the relevant geometry of finite inversive planes and prove some results on automorphisms of associated designs essential for our purpose. In Sect. 6 the geometry and algebra of Sect. 5 will be applied to a Dickson-Ganley unital to obtain crucial information on $\operatorname{Aut}(\mathcal{U})$. In Sect. 7 we solve the isomorphism problem for the Dickson-Ganley unitals, prove the main extension theorem, and determine their (design) automorphism groups.

\section{Dickson semifield planes and Dickson-Ganley unitals}

A finite semifield is an algebraic system that satisfies all axioms of a division ring except the associativity of multiplication. Such a system has various names in the literature, but the term semifield is due to Knuth [21]. Some examples of finite semifields relevant to the study of unitals are the Dickson semifields $[9,10]$, Albert's twisted fields [2], and Albert's generalized twisted fields [3,4].

In more details, a finite semifield $K(+, \cdot)$ is a finite algebraic system with two binary operations, addition and multiplication, which satisfy the following axioms:

1. $K(+)$ is a group with identity 0 .

2. For any $a, b \in S$, if $a b=0$, then $a=0$ or $b=0$.

3. There is an element $1 \in S$ such that for any $a \in S, 1 a=a 1=a$.

4. For any $a, b, c \in S, a(b+c)=a b+a c$ and $(a+b) c=a c+b c$.

The additive group $K(+)$ of $K$ is an elementary Abelian group and so $K(+)$ is isomorphic to $(\mathbb{Z} / p \mathbb{Z})^{N}$ for some prime $p$ (called the characteristic of the semifield) and positive integer $N$. Thus $K$ can also be considered as a vector space over the finite field $\mathbb{F}_{p}$, and $|K|=p^{N}$ (see [21]).

Since a semifield is a particular kind of ternary ring, it can be used to coordinatize a projective plane $\Pi(K)[14]$. Here we follow the notations of Ganley 
[13]. The set of points of $\Pi(K)$ is $\{(z, w) \mid z, w \in K\} \cup\{(m) \mid m \in K\} \cup\{(\infty)\}$ where $\infty$ is a symbol not in $K$. The set of lines of $\Pi(K)$ is $\{[m, k] \mid m, k \in$ $K\} \cup\{[z] \mid z \in K\} \cup\{[\infty]\}$, where $[m, k]=\{(m)\} \cup\{(z, w) \in K \times K \mid m z+w=k\}$, $[z]=\{(\infty)\} \cup\{(z, w) \mid w \in K\}$, and $[\infty]=\{(\infty)\} \cup\{(m) \mid m \in K\}$. Incidence is set-theoretic inclusion. We call such a projective plane a semifield plane. Any semifield plane has the property that $[\infty]$ and $(\infty)$ are respectively a translation line and a translation point [24]. Two semifields $K_{1}\left(+_{1}, \cdot_{1}\right)$ and $K_{2}\left(+_{2}, \cdot 2\right)$ are said to be isotopic if there exists an ordered triple $(A, B, C)$ of additive bijections from $K_{1}$ to $K_{2}$ such that $\left(a \cdot{ }_{1} b\right)^{A}=a^{B} \cdot{ }_{2} b^{C}$ for all $a, b \in K_{1}$. $(A, B, C)$ is called an isotopism from $K_{1}$ to $K_{2}$, and an autotopism if $K_{1}=K_{2}$. In case $A=B=C, K_{1}$ and $K_{2}$ are isomorphic. The geometric significance of isotopy is the following: two semifields coordinatize isomorphic planes if and only if they are isotopic [3, Theorem 6].

We now turn our attention to the Dickson (commutative) semifields $[9,10]$. Consider the finite field $\mathbb{F}_{q}$, where $q=p^{e}$ for an odd prime $p$, and $e>1$. Then there is a non-square element $\delta$ in $\mathbb{F}_{q}$ and a non-identity automorphism $\sigma$ of $\mathbb{F}_{q}$. Let $\mathcal{K}$ be a two-dimensional vector space over $\mathbb{F}_{q}$ with basis elements 1 and $\lambda$. Define multiplication of $\mathcal{K}$ by $(x+\lambda y)(u+\lambda v)=x u+\delta y^{\sigma} v^{\sigma}+\lambda(x v+y u)$. Then $\mathcal{K}(+, \cdot)$ is a commutative semifield where + is the vector space addition of $\mathcal{K}$ over $\mathbb{F}_{q} . \mathcal{K}$ is called a Dickson semifield of order $q^{2}$.

For the same automorphism $\sigma$, different choices of non-squares $\delta$ yield isomorphic semifields ([26], Theorem 1 ; see also [6]). Indeed, the isomorphism is $\theta$ : $\mathcal{K}_{1}\left(\delta_{1}, \sigma\right) \longrightarrow \mathcal{K}_{2}\left(\delta_{2}, \sigma\right)$ defined by $x+\lambda y \mapsto x+\lambda y \mu$ where $\mu=\left(\delta_{1} \delta_{2}^{-1}\right)^{(1 / 2) \sigma^{-1}}$. On the other hand, for any two automorphisms $\sigma_{1}$ and $\sigma_{2}$ and any two nonsquares $\delta_{1}$ and $\delta_{2}, \mathcal{K}_{1}\left(\delta_{1}, \sigma_{1}\right)$ and $\mathcal{K}_{2}\left(\delta_{2}, \sigma_{2}\right)$ are isotopic if and only if $\sigma_{1}=\sigma_{2}$ or $\sigma_{1}=\sigma_{2}{ }^{-1}([26]$, Theorem 2 ; see also [6]).

Consider the projective plane $\Pi(\mathcal{K})$ coordinatized by a Dickson semifield $\mathcal{K}$. By a result of Ganley [12, Theorem 5], any projective plane coordinatized by a finite commutative semifield which has a non-trivial involutory automorphism admits a unitary polarity. In particular, since the Dickson semifield $\mathcal{K}$ admits an involutory automorphism $\alpha: x+\lambda y \mapsto x-\lambda y, \Pi(\mathcal{K})$ admits a unitary polarity $\rho$ given by $(\infty) \leftrightarrow[\infty],(x+\lambda y) \leftrightarrow[x-\lambda y],(x+\lambda y, u+\lambda v) \leftrightarrow$ $[x-\lambda y,-u+\lambda v]$. It is readily verified that the absolute points of $\rho$ are $(\infty)$ and all (affine) points of the form $\left(x+\lambda y,-\frac{1}{2}\left(x^{2}-\delta y^{2 \sigma}\right)+\lambda v\right)$ where $x, y, v \in \mathbb{F}_{q}$ (see [13]). Note that the non-absolute lines not incident with $(\infty)$ are those of the form $\left[m_{1}+\lambda m_{2}, k_{1}+\lambda k_{2}\right]$ where $k_{1} \neq \frac{1}{2}\left(m_{1}{ }^{2}-\delta m_{2}{ }^{2 \sigma}\right)$. As for the lines on $(\infty)$, the absolute line is the line at infinity $[\infty]$, the remaining $q^{2}$ affine lines being non-absolute lines. Denote by $\mathcal{U}=\mathcal{U}(\delta, \sigma)$ the polar unital defined by $\rho$, i.e. the unitary block design (of order $q$ ) whose points are the absolute points of $\rho$ and whose blocks are the non-absolute lines of $\rho$. We call $\mathcal{U}$ a Dickson-Ganley unital.

Lemma 2.1. Let $\sigma \in \operatorname{Aut}\left(\mathbb{F}_{q}\right), \sigma \neq i d$, and $\delta_{1}, \delta_{2}$ be two non-squares in $\mathbb{F}_{q}$. Then the Dickson-Ganley unitals $\mathcal{U}\left(\delta_{1}, \sigma\right)$ and $\mathcal{U}\left(\delta_{2}, \sigma\right)$ are isomorphic. 
Proof. Note that the isomorphism $\widehat{\theta}: \Pi\left(\mathcal{K}\left(\delta_{1}, \sigma\right)\right) \longrightarrow \Pi\left(\mathcal{K}\left(\delta_{2}, \sigma\right)\right)$ induced by $\theta$ defined above maps $\mathcal{U}\left(\delta_{1}, \sigma\right)$ one-one onto $\mathcal{U}\left(\delta_{2}, \sigma\right)$. Indeed, suppose $A=$ $(x+\lambda y, u+\lambda v)$ is an absolute point in $\Pi\left(\mathcal{K}\left(\delta_{1}, \sigma\right)\right)$, then $u=-\frac{1}{2}\left(x^{2}-\delta_{1} y^{2 \sigma}\right)$. Since $-\frac{1}{2}\left(x^{2}-\delta_{2}(y \mu)^{2 \sigma}\right)=-\frac{1}{2}\left(x^{2}-\delta_{1} y^{2 \sigma}\right)=u$, the image of $A$ under $\widehat{\theta}$ is an absolute point in $\Pi\left(\mathcal{K}\left(\delta_{2}, \sigma\right)\right)$.

By the lemma, we may write $\mathcal{U}(\sigma)$ for $\mathcal{U}(\delta, \sigma)$.

The automorphism (collineation) group of a semifield plane is well-known (see for example [21]). We specialize to the Dickson semifield plane $\Pi(\mathcal{K})$, and consider the (design) automorphism group $\operatorname{Aut}(\mathcal{U})$, where $\mathcal{U}$ is a Dickson-Ganley unital embedded in $\Pi(\mathcal{K})$. Let $\operatorname{Col}(\mathcal{U})$ be the subgroup of $\operatorname{Aut}(\mathcal{U})$ consisting of the collineations of $\Pi(\mathcal{K})$ stabilizing $\mathcal{U}$. It is known that $\operatorname{Col}(\mathcal{U})$ is transitive on the affine unital points ([13], Lemma 3 ; see also [1]). We shall prove in Sect. 7 that $\operatorname{Aut}(\mathcal{U})=\operatorname{Col}(\mathcal{U})$, and that $\mathcal{U}\left(\sigma_{1}\right)$ is isomorphic to $\mathcal{U}\left(\sigma_{2}\right)$ if and only if $\sigma_{1}=\sigma_{2}$ or $\sigma_{1}=\sigma_{2}^{-1}$.

\section{The existence of O'Nan configurations in Dickson-Ganley unitals}

In a unital, an O'Nan configuration is a set of four unital lines in general position, intersecting in six unital points. It is known that a classical unital does not contain any O'Nan configuration [22]. In the case of a Dickson-Ganley unital, Ganley has shown that the absolute point $(\infty)$ is never a point of an O'Nan configuration [13, Theorem 1]. However, it is shown in the same paper that if $p$ is a prime with $p \equiv 3(\bmod 4)$, then there exists an integer $r \leq 2 p^{s}-1$ and an odd integer $k$ with $e=r k$, such that the DicksonGanley unital $\mathcal{U}(\sigma)$ of order $q=p^{e}$, where $\sigma: x \mapsto x^{p^{s}}$, admits an O'Nan configuration.

In this section, we extend Ganley's result to the existence of an O'Nan configuration in any Dickson-Ganley unital. Indeed, we prove the existence of an O'Nan configuration on any affine (non-absolute) line through $(\infty)$ (Theorem 3.1). Our strategy is to set up three possibly repeated nonabsolute lines not incident with $(\infty)$ and meeting the given affine line in three distinct affine absolute points, so that these three non-absolute lines are distinct, non-concurrent, and meet each other in absolute points, provided that it is possible to choose a non-square $\delta$ not satisfying certain polynomials. This we prove to be always possible, which means that the four lines and six points under investigation do constitute an O'Nan configuration.

Recall from Sect. 2 that $\operatorname{Col}(\mathcal{U})$ is transitive on the affine absolute points. Since $\operatorname{Col}(\mathcal{U})$ fixes the absolute point $(\infty)$, it is transitive on the affine (non-absolute) lines through $(\infty)$. We have the following theorem:

Theorem 3.1. There exists an O'Nan configuration on any affine line through $(\infty)$ in any Dickson-Ganley unital. 
Proof. By the remark preceding the theorem, we may assume without loss of generality that the given affine line is $[0]$.

Let $P_{i}=\left(x_{i}+\lambda y_{i}, \frac{\delta y_{i}{ }^{2 \sigma}-x_{i}{ }^{2}}{2}+\lambda w_{i}\right)$ and $l_{j}^{\prime}=\left[k_{j}+\lambda l_{j}, \lambda v_{j}\right]$, for $1 \leq i, j \leq 3$. Then the $P_{i}$ 's and $[0] \cap l_{i}^{\prime}$ 's are all absolute points. For all $i \neq j, P_{i} \in l_{j}^{\prime}$ if and only if

$$
\left(x_{i}+\lambda y_{i}\right)\left(k_{j}+\lambda l_{j}\right)+\frac{\delta y_{i}^{2 \sigma}-x_{i}^{2}}{2}+\lambda w_{i}=\lambda v_{j} .
$$

Hence we have, for $i \neq j$, the twelve equations $\left(A_{i j}\right)$ and $\left(B_{i j}\right)$ :

$$
\begin{aligned}
& \left(A_{i j}\right): x_{i} k_{j}+\delta y_{i}{ }^{\sigma} l_{j}{ }^{\sigma}+\frac{\delta y_{i}{ }^{2 \sigma}-x_{i}{ }^{2}}{2}=0, \\
& \left(B_{i j}\right): y_{i} k_{j}+x_{i} l_{j}+w_{i}=v_{j} .
\end{aligned}
$$

Writing $B_{i j}$ 's in matrix form $\mathbf{M x}=\mathbf{b}$, we have

$$
\left(\begin{array}{cccccc}
1 & 0 & 0 & 0 & 0 & -1 \\
0 & 1 & 0 & -1 & 0 & 0 \\
0 & 0 & 1 & 0 & -1 & 0 \\
0 & 0 & -1 & 1 & 0 & 0 \\
-1 & 0 & 0 & 0 & 1 & 0 \\
0 & -1 & 0 & 0 & 0 & 1
\end{array}\right)\left(\begin{array}{c}
v_{1} \\
v_{2} \\
v_{3} \\
w_{1} \\
w_{2} \\
w_{3}
\end{array}\right)=\left(\begin{array}{c}
y_{3} k_{1}+x_{3} l_{1} \\
y_{1} k_{2}+x_{1} l_{2} \\
y_{2} k_{3}+x_{2} l_{3} \\
-\left(y_{1} k_{3}+x_{1} l_{3}\right) \\
-\left(y_{2} k_{1}+x_{2} l_{1}\right) \\
-\left(y_{3} k_{2}+x_{3} l_{2}\right)
\end{array}\right)
$$

It is easy to check that $\operatorname{rank}(\mathbf{M})=5$. We want to show that $\mathbf{b}$ is in $\operatorname{col}(\mathbf{M})$, which is equivalent to having $\mathbf{b}^{T} \mathbf{1}=0$, i.e.

$$
\begin{gathered}
\left(x_{1}-x_{2}\right)\left(l_{2}-l_{3}\right)+\left(x_{3}-x_{2}\right)\left(l_{1}-l_{2}\right)+ \\
\left(y_{1}-y_{2}\right)\left(k_{2}-k_{3}\right)+\left(y_{3}-y_{2}\right)\left(k_{1}-k_{2}\right)=0 .
\end{gathered}
$$

Let $\tau=\sigma^{-1}$. We introduce parameters $\alpha, \beta, \gamma, m$ and specialize as follows:

$$
\begin{aligned}
l_{1}-l_{2}=(m \gamma)^{\tau}, & k_{1}-k_{2}=\gamma, \\
l_{2}-l_{3}=0, & k_{2}-k_{3}=1, \\
l_{3}-l_{1}=(-m \gamma)^{\tau}, & k_{3}-k_{1}=-\gamma-1, \\
x_{3}-x_{2}=2 \alpha, & y_{3}-y_{2}=2 \beta^{\tau} .
\end{aligned}
$$

We shall solve for $x_{i}, y_{i}, k_{i}, l_{i}$ 's in terms of $\alpha, \beta, \gamma$ and $m$, excluding any solutions leading to $P_{i} \in[0]$, for $i=1,2,3$.

By equations $\left(A_{12}\right)$ and $\left(A_{13}\right)$,

$$
x_{1}\left(k_{2}-k_{3}\right)+\delta y_{1}{ }^{\sigma}\left(l_{2}-l_{3}\right)^{\sigma}=0 .
$$

By our specialization, this becomes $x_{1}=0$ and then $\left(A_{12}\right)$ gives $y_{1}=-2 l_{2}$ :

$$
\begin{aligned}
& x_{1}=0, \\
& y_{1}=-2 l_{2} .
\end{aligned}
$$

Since $P_{1} \notin[0], y_{1} \neq 0$, and so $l_{2} \neq 0$.

Similarly, using $\left(A_{21}\right)$ and $\left(A_{23}\right)$, we have

$$
x_{2}\left(k_{3}-k_{1}\right)+\delta y_{2}{ }^{\sigma}\left(l_{3}-l_{1}\right)^{\sigma}=0 .
$$

By our specialization, this becomes $y_{2}{ }^{\sigma}=-\frac{x_{2}}{\delta} \frac{\gamma+1}{m \gamma}$. 
We now choose $m \neq 1$ and let $\gamma=\frac{1}{m-1}$.

Then $y_{2}{ }^{\sigma}=-\frac{x_{2}}{\delta}$. Substituting $y_{2}{ }^{\sigma}$ into $\left(A_{21}\right)$, we obtain

$$
x_{2} k_{1}+\delta\left(-\frac{x_{2}}{\delta}\right) l_{1}^{\sigma}+\frac{\delta^{-1} x_{2}^{2}-x_{2}^{2}}{2}=0 .
$$

Since $P_{2} \notin[0]$ and so $x_{2} \neq 0$, it follows that

$$
\begin{aligned}
& x_{2}=\frac{2 \delta\left(k_{2}-l_{2}{ }^{\sigma}-1\right)}{\delta-1}, \\
& y_{2}^{\sigma}=\frac{-2\left(k_{2}-l_{2}{ }^{\sigma}-1\right)}{\delta-1} .
\end{aligned}
$$

By $\left(A_{31}\right),\left(A_{32}\right)$,

$$
x_{3}\left(k_{1}-k_{2}\right)+\delta y_{3}{ }^{\sigma}\left(l_{1}{ }^{\sigma}-l_{2}{ }^{\sigma}\right)=0 .
$$

So we have $y_{3}{ }^{\sigma}=-\frac{x_{3}}{m \delta}$. Substituting $y_{3}{ }^{\sigma}$ into $\left(A_{32}\right)$, we obtain

$$
x_{3} k_{2}+\left(-\frac{x_{3} l_{2}^{\sigma}}{m}\right)+\frac{1}{2}\left(\frac{x_{3}{ }^{2}}{m^{2} \delta}-x_{3}{ }^{2}\right)=0 .
$$

Since $P_{3} \notin[0]$ and so $x_{3} \neq 0$, it follows that

$$
\begin{aligned}
x_{3} & =\frac{2\left(k_{2} m-l_{2}{ }^{\sigma}\right) m \delta}{m^{2} \delta-1}, \\
y_{3}{ }^{\sigma} & =-\frac{2\left(k_{2} m-l_{2}{ }^{\sigma}\right)}{m^{2} \delta-1} .
\end{aligned}
$$

Then

$$
\begin{aligned}
& x_{3}-x_{2}=2 \delta\left(\frac{m\left(k_{2} m-l_{2}{ }^{\sigma}\right)}{m^{2} \delta-1}-\frac{k_{2}-l_{2}{ }^{\sigma}-1}{\delta-1}\right), \\
& y_{1}-y_{2}=-2 l_{2}+\frac{2\left(k_{2}{ }^{\tau}-l_{2}-1\right)}{\delta^{\tau}-1}, \\
& y_{3}-y_{2}=-\frac{2\left(k_{2}{ }^{\tau} m^{\tau}-l_{2}\right)}{m^{2 \tau} \delta^{\tau}-1}+\frac{2\left(k_{2}^{\tau}-l_{2}-1\right)}{\delta^{\tau}-1} .
\end{aligned}
$$

In terms of $\alpha$ and $\beta$, we obtain:

$$
\begin{aligned}
\beta m+\frac{\alpha}{\delta} & =\frac{\left(k_{2}-l_{2}{ }^{\sigma}-1\right)(m-1)}{\delta-1}, \\
\beta+\frac{\alpha}{\delta} & =-\frac{(m-1)\left(l_{2}{ }^{\sigma}-k_{2} m\right)}{m^{2} \delta-1} .
\end{aligned}
$$

Since $m \neq 1$, this system of linear equations in $k_{2}, l_{2}^{\sigma}$ has a unique solution. Indeed,

$$
l_{2}{ }^{\sigma}=\frac{\beta(m+1)+\alpha m}{(m-1)}+\frac{\alpha}{\delta(m-1)}-\frac{m}{m-1},
$$

and

$$
k_{2}=l_{2}{ }^{\sigma}+\frac{\delta-1}{m-1}\left(\beta m+\frac{\alpha}{\delta}\right)+1 .
$$


Then (3.1) becomes

$$
\begin{aligned}
\alpha^{\sigma}(\gamma m) & -\left(\frac{\beta(m+1)+\alpha m}{(m-1)}+\frac{\alpha}{\delta(m-1)}-\gamma m\right) \\
& +\left(\beta m+\frac{\alpha}{\delta}\right) \frac{1}{m-1}+\beta(\gamma)^{\sigma}=0 .
\end{aligned}
$$

Now choose $m$ further so that it is not fixed by $\sigma$, and $\alpha$ such that $\alpha^{\sigma}-\alpha+1 \neq 0$. Then (3.1) further becomes

$$
\left(\left(\frac{1}{m^{\sigma}-1}\right)-\frac{1}{m-1}\right) \beta+\frac{\left(\alpha^{\sigma}-\alpha+1\right) m}{m-1}=0 .
$$

Since $\sigma$ does not fix $m,(3.1)$ is satisfied by a unique nonzero $\beta$ which is independent of $\delta$. Then we compute $l_{2}^{\sigma}$ and $k_{2}$, and then a set of $x_{i}, y_{i}, l_{i}, k_{i}, v_{i}, w_{i}$ 's depending only on $\alpha, m, \delta$ and $\sigma$ satisfying $\left(A_{i j}\right)$ and $\left(B_{i j}\right)$ 's.

By Lemma 2.1, the validity of the theorem is independent of the choice of $\delta$. It can be seen that each of the expressions (3.2), (3.3) and (3.5) vanishes for at most one value of $\delta$. For example, by (3.4), the expression (3.2) vanishes for at most one $\delta$. But the number of non-squares in $\mathbb{F}_{q}$ is $\frac{q-1}{2} \geq \frac{9-1}{2}=4$. That is, we can always choose a non-square with (3.2), (3.3) and (3.5) non-vanishing. Then, with such a choice, (3.2) does not vanish implies that $P_{2} \notin[0]$ and $P_{2} \neq P_{1} ;(3.3)$ does not vanish implies that $P_{3} \notin[0]$ and $P_{3} \neq P_{1} ;(3.5)$ does not vanish implies that $P_{1} \notin[0]$. Together with the fact that $y_{3}-y_{2}=2 \beta^{\tau} \neq 0$, we conclude that $P_{1}, P_{2}$ and $P_{3}$ are three distinct absolute points not on [0]. Hence $l_{1}^{\prime}=P_{2} \cdot P_{3}, l_{2}^{\prime}=P_{3} \cdot P_{1}, l_{3}^{\prime}=P_{1} \cdot P_{2}$ and any of these lines is distinct from [0]. Moreover, $l_{i}^{\prime} \neq l_{j}^{\prime}$ since $k_{i}-k_{j} \neq 0 \forall i \neq j$. Finally $l_{i}^{\prime} \cap[0]=\left(0, \lambda v_{i}\right)$ are of course absolute points. Thus the four lines $l_{1}^{\prime}, l_{2}^{\prime}, l_{3}^{\prime}$ and [0] and their intersection points form an O'Nan configuration in the Dickson-Ganley unital.

Corollary 3.2. $\operatorname{Aut}(\mathcal{U})$ fixes $(\infty)$.

Proof. If not, take an O'Nan configuration on the affine absolute point $(\infty)^{\Phi}$ for some $\Phi \in \operatorname{Aut}(\mathcal{U})$. Then $\Phi^{-1}$ carries the O'Nan configuration to $(\infty)$, contradicting that fact that there exists no O'Nan configuration on $(\infty)$.

The following result is immediate from Theorem 3.1 and the nonexistence of O'Nan configuration in the classical unital:

Corollary 3.3. Every Dickson-Ganley unital is non-classical.

\section{Wilbrink's condition (II) and Dickson-Ganley unitals}

Throughout this section, let $q=p^{e}$, where $p$ is an odd prime and $e>1, \delta$ be a non-square in $\mathbb{F}_{q}$ and $\sigma: x \mapsto x^{p^{s}}$ a non-identity automorphism of $\mathbb{F}_{q}$, where $1 \leq s<e$. Moreover, let $\mathcal{U}=\mathcal{U}(\sigma)$ denote the polar unital as defined in Sect. 2. In this section, we show that $\mathcal{U}$ is not classical by proving that Wilbrink's second condition does not hold at any absolute point except the point $(\infty)$. 
In [30] Wilbrink characterizes the classical unital by three intrinsic conditions, called (I), (II) and (III). Here we recall the second condition, (II). Given a unital $U$, i.e. a unitary block design of points and lines (blocks) with parameter $2-\left(q^{3}+1, q+1,1\right)$. Wilbrink's condition (II) on $U$ is as follows.

Condition (II). Let $O$ be a point, $l_{1}$ a line through $O$, and $l$ a line not incident with $O$ such that $l_{1}$ meets $l$ at $A$. For any point $B$ on $l_{1}$ which is different from $O$ and $A$, there exists a line $l^{\prime}$ passing through $B$ but not $O$ and intersecting all lines from $O$ which meet $l$.

We call $O$ in Wilbrink's condition (II) a vertex for the condition. We now check which point of $\mathcal{U}$ is a vertex for condition (II). First consider the case where $O=(\infty)$. Recall from Sect. 2 that any affine line on $(\infty)$ is of the form $[x+\lambda y]$ with the absolute points on it given by $(x+\lambda y, u+\lambda v)$ where $u=-\frac{1}{2}\left(x^{2}-\delta y^{2 \sigma}\right)$, and that any line not incident with $(\infty)$ is of the form $\left[m_{1}+\lambda m_{2}, k_{1}+\lambda k_{2}\right]$, with $k_{1} \neq \frac{1}{2}\left(m_{1}{ }^{2}-\delta m_{2}{ }^{2 \sigma}\right)$ if and only if the line is non-absolute.

Lemma 4.1. Let $l=\left[m_{1}+\lambda m_{2}, k_{1}+\lambda k_{2}\right]$ be a non-absolute line, and $\mathcal{N}$ the set of lines on $(\infty)$ intersecting $l$ at absolute points. For any $k_{2}^{\prime} \in \mathbb{F}_{q}$, the line $l^{\prime}=\left[m_{1}+\lambda m_{2}, k_{1}+\lambda k_{2}^{\prime}\right]$ intersects all lines in $\mathcal{N}$ at absolute points. Conversely, any line intersecting all lines in $\mathcal{N}$ at absolute points is of this form.

Proof. Suppose $(x+\lambda y, u+\lambda v)$ is a unital point on $l$, so that

$$
\begin{aligned}
m_{1} x+\delta m_{2}{ }^{\sigma} y^{\sigma}+u & =k_{1}, \\
m_{2} x+m_{1} y+v & =k_{2}, \\
u & =-\frac{1}{2}\left(x^{2}-\delta y^{2 \sigma}\right) .
\end{aligned}
$$

Using (4.1)-(4.3), it is readily verified that $l^{\prime}$ meets $[x+\lambda y]$ at the unital point $\left(x+\lambda y, u+\lambda\left(k_{2}^{\prime}-k_{2}+v\right)\right)$.

Note that the $q$ lines $\left[m_{1}+\lambda m_{2}, k_{1}+\lambda k_{2}^{\prime}\right]$, where $k_{2}^{\prime} \in \mathbb{F}_{q}$, intersect $\mathcal{N}$ at distinct absolute points. If there is another line intersecting the lines of $\mathcal{N}$ at absolute points, it will give rise to an O'Nan configuration containing $(\infty)$, contradicting Ganley's result [13, Theorem 1]. This proves the converse.

The following Corollary is immediate from Lemma 4.1. Note that by Lemma 4.1, if $k_{1} \neq \frac{1}{2}\left(m_{1}{ }^{2}-\delta m_{2}{ }^{2 \sigma}\right)$, then the $q$ lines $\left[m_{1}+\lambda m_{2}, k_{1}+\lambda k_{2}\right]$, where $k_{2} \in \mathbb{F}_{q}$, are parallel in the sense that they intersect the same lines through $(\infty)$ at distinct absolute points. In this sense, we say that $(\infty)$ is a vertex of Wilbrink's condition (II) in strong form.

Corollary 4.2. $(\infty)$ is a vertex for Wilbrink's condition (II) in strong form.

As the $\lambda$-free part and the $\lambda$-part of the coordinates of unital points on a given non-absolute line missing $(\infty)$ are not related as simply as those on a line through $(\infty)$, we suspect that any other choice of an absolute point might fail to be a vertex for Wilbrink's condition (II). This is indeed the case: 
Theorem 4.3. Any affine absolute point is not a vertex for Wilbrink's condition (II).

Proof. Since $\operatorname{Col}(\mathcal{U})$ is transitive on the affine absolute points, it suffices to prove that the absolute point $O=(0,0)$ is not a vertex for Wilbrink's condition (II).

Consider the absolute points $O:=(0,0), A:=\left(1,-\frac{1}{2}+\lambda\right)$ and $B:=(2 \lambda, 2 \delta-\lambda)$ on $l_{1}:=\left[\frac{1}{2}-\lambda, 0\right]$. Let $l$ be [1], a non-absolute line on $A$. The lines through $O$ meeting $l_{1}$ at an absolute point constitute the set $\mathcal{N}:=\{[0]\} \cup\left\{\left[\frac{1}{2}-\lambda t, 0\right], t \in\right.$ $\left.\mathbb{F}_{q}\right\}$. Suppose there is a line $l^{\prime}$ on $B$ meeting each line in $\mathcal{N}$ at an absolute point. Then $l^{\prime} \in\{(2 \lambda, 2 \delta-\lambda) \cdot(\infty)\} \cup\left\{(2 \lambda, 2 \delta-\lambda) \cdot(0, \lambda w) \mid w \in \mathbb{F}_{q} \backslash\{0\}\right\}$ because the absolute points on $[0]$ are $(\infty)$ and $(0, \lambda w)$ where $w \in \mathbb{F}_{q}$. We aim to show that each of these lines meets a line in $\mathcal{N}$ at a non-absolute point.

First note that $(2 \lambda, 2 \delta-\lambda) \cdot(\infty)=[2 \lambda]$. It intersects $\left[\frac{1}{2}, 0\right]$ at $(2 \lambda,-\lambda)$ which is not an absolute point. Therefore $l^{\prime} \neq[2 \lambda]$.

Now for each nonzero $w \in \mathbb{F}_{q},(2 \lambda, 2 \delta-\lambda) \cdot(0, \lambda w)=\left[\frac{w+1}{2}-\lambda, \lambda w\right]$. Suppose $\left[\frac{w+1}{2}-\lambda, \lambda w\right]$ meets $\left[\frac{1}{2}-\lambda t, 0\right]$ at $(x+\lambda y, u+\lambda v)$ where $t \neq 1$. Then

$$
\begin{gathered}
\left(\frac{w+1}{2}-\lambda\right)(x+\lambda y)+(u+\lambda v)=\lambda w, \\
\left(\frac{1}{2}-\lambda t\right)(x+\lambda y)+(u+\lambda v)=0 .
\end{gathered}
$$

Suppose further this is an absolute point. Then we have the system of equations:

$$
\begin{aligned}
\frac{w+1}{2} x-\delta y^{\sigma}+u & =0, \\
-x+\frac{w+1}{2} y+v & =w, \\
\frac{x}{2}-\delta y^{\sigma} t^{\sigma}+u & =0, \\
\frac{y}{2}-t x+v & =0, \\
u & =\frac{\delta y^{2 \sigma}-x^{2}}{2} .
\end{aligned}
$$

We aim to show that for each $w \neq 0$, there exists $t \neq 1$ such that there is no solution to this system (4.4)-(4.8).

Subtracting (4.6) from (4.4) and then (4.7) from (4.5), we obtain

$$
\begin{aligned}
w x & =-2 \delta y^{\sigma}\left(t^{\sigma}-1\right), \\
2(1-t) x & =w(y-2) .
\end{aligned}
$$

Eliminating $u$ and then $x$ in (4.8) by (4.4) and (4.9) respectively, we obtain

$$
y^{2 \sigma}\left(w^{2}-4 \delta\left(t^{\sigma}-1\right)^{2}\right)=2 y^{\sigma} w\left(t^{\sigma} w+t^{\sigma}-1\right) .
$$


Note that $y^{\sigma} \neq 0$. Otherwise, since $w \neq 0, x=0$ by (4.9) and so $y=2$ by (4.10), which is a contradiction. Canceling $y^{\sigma}$ gives

$$
y^{\sigma}=\frac{2 w\left(t^{\sigma} w+t^{\sigma}-1\right)}{w^{2}-4 \delta\left(t^{\sigma}-1\right)^{2}} .
$$

In case $w \neq-1$, take $t=\frac{1}{w^{\sigma^{-1}}+1}$. Then $t^{\sigma} w+t^{\sigma}-1=0$ and by (4.11), $y^{\sigma}=0$, which we have shown to be impossible. So suppose $w=-1$. Substituting $w=-1$ in (4.11), we obtain

$$
y^{\sigma}=\frac{2}{1-4 \delta\left(t^{\sigma}-1\right)^{2}} .
$$

Eliminating $x$ in (4.9) by (4.10), we obtain $(y-2) w^{2}=4 y^{\sigma} \delta(t-1)\left(t^{\sigma}-1\right)$. Substituting $y^{\sigma}$ given by (4.12), this becomes

$$
y-2=\frac{8 \delta(t-1)\left(t^{\sigma}-1\right)}{1-4 \delta\left(t^{\sigma}-1\right)^{2}} .
$$

Applying $\sigma^{-1}$ to both sides of (4.13), we obtain

$$
y^{\sigma^{-1}}-2=\frac{8 \delta^{\sigma^{-1}}\left(t^{\sigma^{-1}}-1\right)(t-1)}{1-4 \delta^{\sigma^{-1}}(t-1)^{2}} .
$$

Combining (4.13) and (4.14) this gives

$$
\frac{\delta^{\sigma^{-1}}(t-1)}{1-4 \delta^{\sigma^{-1}}(t-1)^{2}}=\left(\frac{\delta^{\sigma^{-1}}(t-1)}{1-4 \delta^{\sigma^{-1}}(t-1)^{2}}\right)^{\sigma} .
$$

Since there are at most $p^{s}$ solutions $z \in \mathbb{F}_{q}$ to $z=z^{\sigma}$, there are at most $2 p^{s}<p^{e}-1=q-1$ solutions $t$ to (4.15) as $p \geq 3$ and $e>s$. Hence, there exists $t \neq 1 \in \mathbb{F}_{q}$ for which there is no solution to the system (4.4)-(4.8). In other words, there is a line in $\mathcal{N}$ which intersects $[-\lambda,-\lambda]$ at a non-absolute point.

By Corollary 4.2 and Theorem 4.3, we obtain the same result as in Section 3:

Corollary 4.4. $\operatorname{Aut}(\mathcal{U})$ fixes $(\infty)$.

The following result of Sect. 3 is also immediate from Theorem 4.3 and Wilbrink's characterization of a classical unital [30]:

Corollary 4.5. Every Dickson-Ganley unital is non-classical.

\section{Inversive plane geometry}

The point $(\infty)$ of a Dickson-Ganley unital $\mathcal{U}$ is special. In particular, it satisfies Wilbrink's condition (II) in strong form (Corollary 4.2). This makes it possible to construct from $\mathcal{U}$ a design $\mathcal{S}$ which turns out to be isomorphic to the residual of a classical inversive plane. The structure of $\operatorname{Aut}(\mathcal{S})$, the automorphism group of $\mathcal{S}$, plays a key role in our study on the structure of $\operatorname{Aut}(\mathcal{U})$, 
the automorphism group of $\mathcal{U}$. In this section, we recall the geometry of inversive planes needed for our purpose and establish some lemmas which allow us to study $\mathcal{S}$ and $\operatorname{Aut}(\mathcal{S})$ in some details.

An inversive plane is a set of points with distinguished subsets of the points, called circles satisfying the following axioms:

(IP1) Any three distinct points are contained in exactly one common circle.

(IP2) If $P$ and $Q$ are points and if $c$ is a circle containing $P$ but not $Q$, then there is a unique circle $d$ such that $P, Q \in d$ and $c \cap d=\{P\}$.

(IP3) There are four points not on a common circle.

We are concerned only with the finite inversive planes. These are exactly the class of 3 -designs with parameters $\left(n^{2}+1, n+1,1\right)$ with $n \geq 2$. (We refer to Dembowski [7] for standard results on finite inversive planes.) We call $n$ the order of a finite inversive plane.

Let $\mathcal{I}$ be a finite inversive plane of order $n$. A bundle of circles of $\mathcal{I}$, denoted by $[P, Q]$, is the set of all circles through the points $P, Q$ of $\mathcal{I}$, with $P \neq Q$. The points $P, Q$ are called the carriers of the bundle. The following lemma studies how a circle not belonging to a bundle meets the circles of the bundle. Note that by axiom (IP1), two distinct circles intersect in 0,1 , or 2 points. Accordingly we say that the two circles are disjoint, tangent, or intersecting, respectively.

Lemma 5.1. Let $P, Q$ be two distinct points not on a circle $c$ in an inversive plane $\mathcal{I}$ of order $n$. Then one of the following statements is true:

1. $n$ is odd, and $c$ is respectively disjoint from $(n+1) / 2$, tangent to 0 , and intersecting $(n+1) / 2$, circles of $[P, Q]$.

2. $n$ is odd, and $c$ is respectively disjoint from $(n-1) / 2$, tangent to 2 , and intersecting $(n-1) / 2$, circles of $[P, Q]$.

3. $n$ is even, and $c$ is respectively disjoint from $n / 2$, tangent to 1 , and intersecting $n / 2$, circles of $[P, Q]$.

4. $n$ is even, and $c$ is tangent to all $n+1$ circles of $[P, Q]$.

Proof. This is (2.9) and (2.10) of [8] and simple counting from axioms.

We shall also need to consider another type of circle set. A flock is a set $\mathcal{F}$ of mutually disjoint circles in $\mathcal{I}$ which partitions the points of $\mathcal{I}$ except two distinct points $P$ and $Q$. These points are again called the carriers of the flock. The existence of flocks is not as clear as that of bundles, and a flock need not be determined by its carriers. However, in an egglike inversive plane, it is known that every flock is uniquely determined by its carriers, as we recall below.

Let $O$ be an ovoid in $P G(3, q), q>2$, i.e. a set of $q^{2}+1$ points in general position (no three of which are collinear) in $P G(3, q)$. An example is the elliptic quadric which has canonical form $f\left(x_{0}, x_{1}\right)+x_{2} x_{3}=0$, where $f$ is an irreducible quadratic form. As in the case of an elliptic quadric, at each point of an ovoid there is a unique tangent plane, and all other (secant) planes meet the ovoid in an oval, i.e. a set of $q+1$ points in general position (see [15]). Given an ovoid 
$O$, an inversive plane $\mathcal{I}(O)$ of order $q$ can be constructed as follows: points of $\mathcal{I}(O)$ are the points of $O$, circles are the secant plane intersections of $O$, and incidence is set-theoretic. An inversive plane is called egglike if it is isomorphic to $\mathcal{I}(O)$ for some ovoid $O$; it is called classical if $O$ is an elliptic quadric. An inversive plane is called Miquelian if it satisfies the Theorem of Miquel. It is known that an inversive plane is classical if and only if it is Miquelian [29]. Since we shall not need the Theorem of Miquel, we do not state it here.

Consider now an external line $l$ of $O$, i.e. a line not meeting $O$. The planes on $l$ give rise to a flock of $\mathcal{I}(O)$ with carriers $P, Q$, as follows: the circles are the intersections of $O$ by the secant planes on $l$, and the carriers are intersections of $O$ by the tangent planes on $l$. Such a flock is called linear (see for example $[28])$. Concerning linear flocks, there is the following result ([23,27]; see also [11]):

Theorem 5.2. Every flock of an egglike inversive plane is linear.

Since in $P G(3, q)$, any two tangent planes of an ovoid $O$ meet in an external line of $O$, every flock of an egglike inversive plane is uniquely determined by its carriers. We denote such a flock by $\mathcal{F}(P, Q)$ with carriers $P$ and $Q$.

We are almost ready to prove the main result of this section. Let $\mathcal{I}$ be an egglike inversive plane. Let $X$ be a point of $\mathcal{I}$, and consider the residual $\mathcal{I}^{X}$ of $\mathcal{I}$ at $X$. Recall that given any design $S$ and a point $X$ of $S$, the residual (design) $S^{X}$ at $X$ is obtained by deleting the point $X$ and all blocks containing $X$. We are going to show that the automorphism group $\operatorname{Aut}\left(\mathcal{I}^{X}\right)$ is isomorphic to the subgroup $\operatorname{Aut}(\mathcal{I})_{X}$ of the automorphism group $\operatorname{Aut}(\mathcal{I})$ fixing $X$.

We shall need the result that any egglike inversive plane satisfies the Bundle Theorem $[7,29]$. The latter theorem is as follows:

Bundle Theorem. Let $\left\{P_{i}, Q_{i} \mid i=0,1,2,3\right\}$ be a set of points in an inversive plane $\mathcal{I}$, where $P_{i}, i=0,1,2,3$, are distinct. Let $c_{0}, c_{1}, c_{2}, c_{3}$ be circles in $\mathcal{I}$ such that $c_{i} \cap c_{i+1}=\left\{P_{i}, Q_{i}\right\}$, with subscripts taken mod 4 . Then $P_{0}, Q_{0}, P_{2}, Q_{2}$ are on a common circle if and only if $P_{1}, Q_{1}, P_{3}, Q_{3}$ are on a common circle.

We call the configuration (see Fig. 1) described in the Bundle Theorem a B-configuration.

The following lemma proves that in an egglike inversive plane, any four points on a circle can be completed to a B-configuration.

Lemma 5.3. Consider an egglike inversive plane of order $q \geq 4$. Let $P_{0}$, $Q_{0}, P_{3}, Q_{3}, X$ be five distinct points on a circle $c_{0}$. Then there exist points $P_{1}, Q_{1}, P_{2}, Q_{2}$, with only $P_{2}$ and $Q_{2}$ not necessarily distinct, and five distinct circles $c_{1}, c_{2}, \cdots, c_{5}$ missing $X$, such that $P_{0}, Q_{0}, P_{1}, Q_{1} \in c_{1}, P_{1}, Q_{1}, P_{2}$, $Q_{2} \in c_{2}, P_{2}, Q_{2}, P_{3}, Q_{3} \in c_{3}, P_{1}, Q_{1}, P_{3}, Q_{3} \in c_{4}$ and $P_{0}, Q_{0}, P_{2}, Q_{2} \in c_{5}$.

Proof. By axiom (IP3), there is a circle $c_{1} \neq c_{0}$ in $\left[P_{0}, Q_{0}\right]$. Consider the circle $c_{1}$ and the bundle $\left[P_{3}, Q_{3}\right]$. Since case (4) of Lemma 5.1 does not happen, there is a circle $c_{3} \in\left[P_{3}, Q_{3}\right], c_{3} \neq c_{0}$, such that $c_{3}$ does not meet $c_{1}$, and there is a circle $c_{4} \in\left[P_{3}, Q_{3}\right], c_{4} \neq c_{0}$, such that $c_{4}$ meets $c_{1}$ at two distinct two points, 


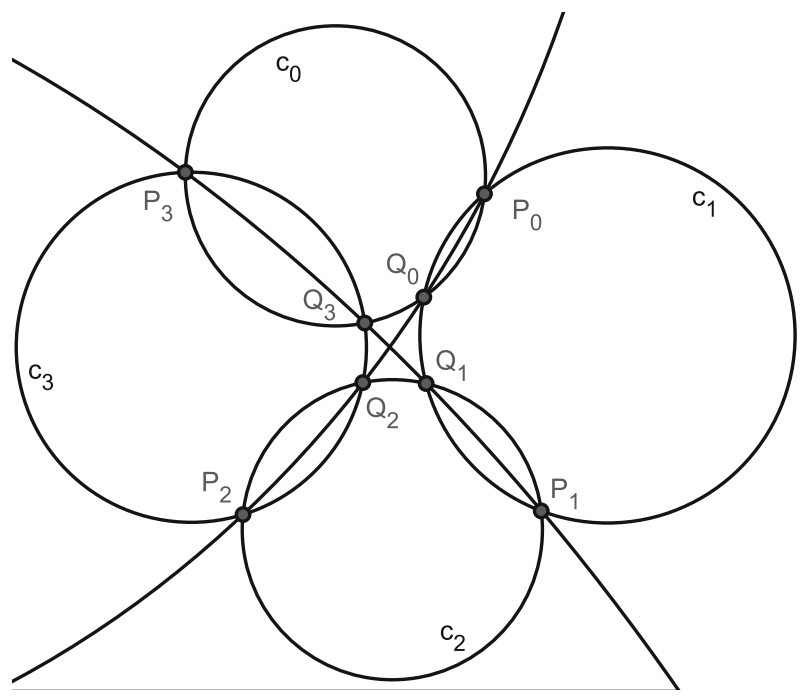

FiguRe 1 B-configuration in $\mathcal{I}$ when $P_{i}$ and $Q_{i}$ are all distinct

say $P_{1}, Q_{1}$. Indeed, in both cases, $\min \{(5-1) / 2,4 / 2\}=2$, by Lemma 5.1. Consider now the circle $c_{3}$ and the bundle $\left[P_{1}, Q_{1}\right]$. By Lemma 5.1, there are at least $\min \{2+(5-1) / 2,(5+1) / 2,1+4 / 2\}=3$ circles in $\left[P_{1}, Q_{1}\right]$ meeting $c_{3}$ at one or two distinct points. Hence there is a circle $c_{2} \in\left[P_{1}, Q_{1}\right]$ different from $c_{4}$ and missing $X$ such that $c_{2}$ meets $c_{3}$ at one or two new distinct points, say $P_{2}, Q_{2}$, with $P_{2}$ and $Q_{2}$ not necessarily distinct. By the Bundle Theorem, $P_{0}, Q_{0}, P_{2}, Q_{2}$ are on a circle, say $c_{5}$.

We are now ready to prove the main result of this section.

Theorem 5.4. Let $\mathcal{I}$ be an egglike inversive plane of order $q$. For any point $X$ of $\mathcal{I}, \operatorname{Aut}\left(\mathcal{I}^{X}\right)$ is isomorphic to $\operatorname{Aut}(\mathcal{I})_{X}$.

Proof. Any element in $\operatorname{Aut}(\mathcal{I})_{X}$ induces an element in $\operatorname{Aut}\left(\mathcal{I}^{X}\right)$ by restriction. On the other hand, given $f \in \operatorname{Aut}\left(\mathcal{I}^{X}\right)$, let $\tilde{f}$ be the extension of $f$ to $\mathcal{I}$ by setting $X^{\tilde{f}}=X$. To show that $\tilde{f}$ defines an element in $\operatorname{Aut}(\mathcal{I})_{X}$, it suffices to show that $\tilde{f}$ maps any circle on $X$ to a circle on $X$.

Since the case when $q=3$ is elementary and is readily verified, we consider the case when $q \geq 4$. Let $c$ be a circle on $X$, and let $P_{0}, Q_{0}, P_{3}, Q_{3}$ be any four distinct points on $c \backslash\{X\}$. By Lemma 5.3, there is a B-configuration containing $P_{0}, Q_{0}, P_{3}, Q_{3}$, and none of its circles, except $c$, are on $X$. Thus the five circles which are not on $X$ are mapped by $\tilde{f}$ to circles not on $X$. By the Bundle Theorem, $P_{0}{ }^{\tilde{f}}, Q_{0}{ }_{0}^{\tilde{f}}, P_{3}{ }^{\tilde{f}}, Q_{3}{ }^{\tilde{f}}$ are on a common circle, say $d$. Note that $d$ is a circle on $X$. For, if $d$ does not pass through $X$, then $d^{\tilde{f}^{-1}}$ is a circle on $P_{0}, Q_{0}, P_{3}, Q_{3}$ but not $X$, contradicting axiom(IP1). Now $d$ is determined by $P_{0} \tilde{f}, Q_{0} \tilde{f}, P_{3} \tilde{f}$, and since $Q_{3}$ is arbitrary, $\tilde{f}$ maps $c$ to $d$.

It is clear that the above defines an isomorphism between the two groups. 


\section{Classical Inversive Plane and Dickson-Ganley unitals}

We return to the Dickson-Ganley unitals. Consider a Dickson unital $\mathcal{U}=\mathcal{U}(\sigma)$ defined by the polarity $\rho$ in a Dickson semifield plane $\Pi(\mathcal{K}(\delta, \sigma))$ as given in Sect. 2. We shall construct from $\mathcal{U}$ a design $\mathcal{S}$ with respect to the special point $(\infty)$. Since $(\infty)$ satisfies Wilbrink's condition (II) in strong form, we can follow the construction of $\mathcal{S}$ from $\mathcal{U}$ as in Wilbrink [30] (see also [5] for further development).

The design $\mathcal{S}=(\mathcal{P}, \mathcal{B}, \mathbf{I})$ is defined as follows. The point set $\mathcal{P}$ consists of the $q^{2}$ unital lines on $(\infty)$. As for the block set $\mathcal{B}$, we introduce an equivalence relation $\sim$ on the unital lines missing $(\infty)$ by $[m, k] \sim\left[m^{\prime}, k^{\prime}\right]$ if and only if $m=m^{\prime}$ and $k-k^{\prime}$ has no $\lambda$-free part. Denote by $\langle[m, k]\rangle$ the equivalence class of $[m, k]$. Thus, $\langle[m, k]\rangle$ consists of the $q$ lines $\left[m, k^{\prime}\right]$ where $k^{\prime}-k=\lambda k_{2}$ with $k_{2} \in \mathbb{F}_{q}$, and the $\lambda$-free part $k_{1}$ of $k$ satisfies $k_{1} \neq \frac{1}{2} m_{1}{ }^{2}-\frac{1}{2} \delta m_{2}{ }^{2 \sigma}$, i.e. the parallel lines described by Lemma 4.1. It follows that the unital lines on each non-affine non-absolute point $(m)$ are partitioned into $q-1$ equivalence classes. There is therefore a total of $q^{2}(q-1)$ equivalence classes and these are the elements of the block set $\mathcal{B}$. We define $[z] \mathbf{I}\langle[m, k]\rangle$ if and only if $[z]$ meets $[m, k]$ at an absolute point. By Lemma $4.1, \mathbf{I}$ is well-defined. Furthermore, since the unital lines joining an affine absolute point on $\left[z_{1}\right]$ to each of the $q$ affine absolute points on $\left[z_{2}\right]$ meet $[\infty]$ at different points, there are $q$ distinct blocks through any two points of $\mathcal{S}$. Thus $\mathcal{S}$ is a $2-\left(q^{2}, q+1, q\right)$ design. We call the blocks of $\mathcal{S}$ circles. Note that the parameters of $\mathcal{S}$ are exactly those of a residual design of an inversive plane of order $q$. Indeed, we shall prove below the main result of this section, namely, that $\mathcal{S}$ is the residual of a classical inversive plane.

To this end, consider the inversive plane $\mathscr{I}=(\mathcal{X}, \mathcal{C})$ whose points are points of the projective line $P G\left(1, q^{2}\right)$ and whose circles are all sublines $P G(1, q)$ in $P G\left(1, q^{2}\right) . \mathscr{I}$ is Miquelian (see [16]) and hence classical.

We describe the circle set $\mathcal{C}$. Let

$$
c_{a_{0}, a_{1}, b}=\left\{[z, w] \in P G\left(1, q^{2}\right) \mid a_{0} z^{q+1}+b z^{q} w+b^{q} z w^{q}+a_{1} w^{q+1}=0\right\} .
$$

Lemma 6.1. $\mathcal{C}=\left\{c_{a_{0}, a_{1}, b} \mid a_{0}, a_{1} \in \mathbb{F}_{q}, b \in \mathbb{F}_{q^{2}} ; a_{0}, a_{1}, b\right.$ not all zeros; $a_{0} a_{1}-$ $\left.b^{q+1} \neq 0\right\}$.

Proof. By Lemmas 5.1 and 6.2 of [15], $\mathcal{C}$ is the set of Hermitian varieties in $P G\left(1, q^{2}\right)$ which are non-degenerate, i.e. non-singular. If $F\left(X_{0}, X_{1}\right)=$ $a_{0} X_{0}{ }^{q+1}+b X_{0}^{q} X_{1}+b^{q} X_{0} X_{1}^{q}+a_{1} X_{1}^{q+1}$, then $\frac{\partial F}{\partial X_{0}}=a_{0} X_{0}^{q}+b^{q} X_{1}^{q}$ and $\frac{\partial F}{\partial X_{1}}=b X_{0}^{q}+a_{1} X_{1}^{q}$, so that a point of $c_{a_{0}, a_{1}, b}$ is singular if and only if the system

$$
\left(\begin{array}{cc}
a_{0} & b^{q} \\
b & a_{1}
\end{array}\right)\left(\begin{array}{l}
X_{0}^{q} \\
X_{1}^{q}
\end{array}\right)=\left(\begin{array}{l}
0 \\
0
\end{array}\right)
$$

has non-trivial solution. The result follows. 
Let $X=[1,0] \in \mathcal{X}$. Then the residual $\mathscr{I}^{X}$ is $\left(\mathcal{X}^{\prime}, \mathcal{C}^{\prime}\right)$, where

$$
\mathcal{X}^{\prime}=\left\{[z, 1] \in P G\left(1, q^{2}\right) \mid z \in \mathbb{F}_{q^{2}}\right\}
$$

and

$$
\mathcal{C}^{\prime}=\left\{c_{a_{0}, a_{1}, b} \mid a_{0}, a_{1} \in \mathbb{F}_{q}, b \in \mathbb{F}_{q^{2}} ; a_{0} \neq 0 ; a_{0} a_{1} \neq b^{q+1}\right\} .
$$

We claim that $\mathcal{S}$ and $\mathscr{I}^{X}$ are isomorphic designs.

Let $\kappa \in \mathbb{F}_{q^{2}}$ be a zero of $X^{2}-\delta \in \mathbb{F}_{q}[X]$. Then $\delta^{q-1}=\kappa^{2(q-1)}=1$, so that

$$
\kappa^{q-1}=-1 \text {. }
$$

Now let $H: \mathcal{S} \rightarrow \mathscr{I}^{X}$ be defined by

$$
\begin{aligned}
{[x+\lambda y] } & \mapsto\left[x+\kappa y^{\sigma}, 1\right], \\
\left\langle\left[m_{1}+\lambda m_{2}, k_{1}+\lambda k_{2}\right]\right\rangle & \mapsto c_{-1,-2 k_{1}, m_{1}-\kappa m_{2} \sigma} .
\end{aligned}
$$

Theorem 6.2. $H$ is an isomorphism.

Proof. Recall that any affine unital line is given by $\left[m_{1}+\lambda m_{2}, k_{1}+\lambda k_{2}\right]$, with $2 k_{1} \neq m_{1}{ }^{2}-\delta m_{2}{ }^{2 \sigma}$. Since $\kappa^{q-1}=-1$, and $m_{1}, m_{2} \in \mathbb{F}_{q}$,

$$
\begin{aligned}
2 k_{1} & \neq m_{1}{ }^{2}-\delta m_{2}{ }^{2 \sigma}=\left(m_{1}-\kappa m_{2}{ }^{\sigma}\right)\left(m_{1}+\kappa m_{2}{ }^{\sigma}\right) \\
& =\left(m_{1}-\kappa m_{2}{ }^{\sigma}\right)\left(m_{1}-\kappa^{q} m_{2}{ }^{\sigma}\right)=\left(m_{1}-\kappa m_{2}{ }^{\sigma}\right)\left(m_{1}-\kappa m_{2}{ }^{\sigma}\right)^{q} \\
& =\left(m_{1}-\kappa m_{2}{ }^{\sigma}\right)^{q+1} .
\end{aligned}
$$

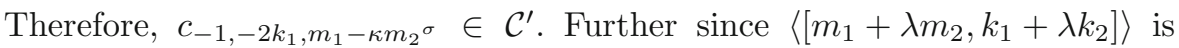
independent of $k_{2}, H$ is a well defined map.

We next check that $H$ preserves incidence. Suppose $[x+\lambda y]$ meets $\left[m_{1}+\right.$ $\left.\lambda m_{2}, k_{1}+\lambda k_{2}\right]$ at $\left(x+\lambda y,-\frac{1}{2} x^{2}+\frac{1}{2} \delta y^{2 \sigma}+\lambda v\right)$. Then

$$
\left\{\begin{aligned}
m_{1} x+\delta m_{2}{ }^{\sigma} y^{\sigma}-\frac{1}{2} x^{2}+\frac{1}{2} \delta y^{2 \sigma} & =k_{1}, \\
m_{2} x+m_{1} y+v & =k_{2} .
\end{aligned}\right.
$$

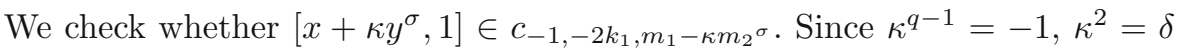
and $x, y, m_{1}, m_{2} \in \mathbb{F}_{q}$,

$$
\begin{aligned}
- & 1\left(x+\kappa y^{\sigma}\right)^{q+1}+\left(m_{1}-\kappa m_{2}{ }^{\sigma}\right)\left(x+\kappa y^{\sigma}\right)^{q}+\left(m_{1}-\kappa m_{2}{ }^{\sigma}\right)^{q}\left(x+\kappa y^{\sigma}\right)-2 k_{1} \\
& =\left(x+\kappa y^{\sigma}\right)\left(x+\kappa^{q} y^{\sigma}\right)+\left(m_{1}-\kappa m_{2}{ }^{\sigma}\right)\left(x+\kappa^{q} y^{\sigma}\right)+\left(m_{1}-\kappa^{q} m_{2}{ }^{\sigma}\right)\left(x+\kappa y^{\sigma}\right)-2 k_{1} \\
& =\left(x+\kappa y^{\sigma}\right)\left(x-\kappa y^{\sigma}\right)+\left(m_{1}-\kappa m_{2}{ }^{\sigma}\right)\left(x-\kappa y^{\sigma}\right)+\left(m_{1}+\kappa m_{2}{ }^{\sigma}\right)\left(x+\kappa y^{\sigma}\right)-2 k_{1} \\
& =\left(x^{2}-\delta y^{2 \sigma}\right)+2 m_{1} x+2 \delta m_{2}{ }^{\sigma} y^{\sigma}-2 k_{1} .
\end{aligned}
$$

By the first equation of (6.2), the above expression equals zero and hence $\left[x+\kappa y^{\sigma}, 1\right] \in c_{-1,-2 k_{1}, m_{1}-\kappa m_{2}{ }^{\sigma}}$.

Clearly, $H$ is an injection on the points of $\mathcal{S}$. Since $H$ preserves incidence, is it also an injection on the circles of $\mathcal{S}$. Since $\mathcal{S}$ and $\mathscr{I}^{X}$ have the same numbers of points and circles, $H$ is a bijection, and hence an isomorphism.

By [16], the automorphism group of a Miquelian inversive plane of order $q$ is given by $P \Gamma L\left(2, q^{2}\right)$. Thus,

$\operatorname{Aut}(\mathscr{I})=\left\{\varphi_{a, b, c, d, \epsilon} \mid a, b, c, d \in \mathbb{F}_{q^{2}}, a d-b c \neq 0, \epsilon \in \operatorname{Aut}\left(\mathbb{F}_{q^{2}}\right)\right\} \cong P \Gamma L\left(2, q^{2}\right)$, 
where

$$
\varphi_{a, b, c, d, \epsilon}: P G\left(1, q^{2}\right) \rightarrow P G\left(1, q^{2}\right)
$$

is defined by

$$
\varphi_{a, b, c, d, \epsilon}:[z, w] \mapsto\left[\left(z^{\epsilon}, w^{\epsilon}\right)\left(\begin{array}{ll}
a & c \\
b & d
\end{array}\right)\right] .
$$

In view of Theorem 6.2, $H$ induces a group isomorphism

$$
h: \operatorname{Aut}(\mathcal{S}) \rightarrow \operatorname{Aut}\left(\mathscr{I}^{X}\right)
$$

given by

$$
H \Psi^{h}=\Psi H
$$

for any $\Psi \in \operatorname{Aut}(\mathcal{S})$.

We now apply Theorem 5.4 to obtain the following result which plays a key role in our study of $\operatorname{Aut}(\mathcal{U})$ in the next section. Let $Y=[0,1]=[0]^{H} \in \mathcal{X}^{\prime}$.

Theorem 6.3. $\operatorname{Aut}\left(\mathscr{I}^{X}\right)_{Y}=\left\{\varphi_{a, 0,0,1, \epsilon}: a \in \mathbb{F}_{q}^{*}, \epsilon \in \operatorname{Aut}\left(\mathbb{F}_{q^{2}}\right)\right\}$.

Proof. Since $\mathscr{I}$ is classical, hence egglike, Theorem 5.4 applies and we have $\operatorname{Aut}\left(\mathscr{I}^{X}\right)=\operatorname{Aut}(\mathscr{I})_{X}$. By (6.3), if $\varphi_{a, b, c, d, \epsilon}$ fixes $X=[1,0]$, then $c=0$. Furthermore, if it fixes $Y=[0,1]$, then $b=0$. Since $\varphi_{a, b, c, d, \epsilon}$ and $\varphi_{a / d, 0,0,1, \epsilon}$ are the same map, the result follows.

Consider now the $q-1$ circles of $\mathcal{S}$ which arise from the partition into equivalence classes of the unital lines on the non-absolute point $(m)$. The point of $\mathcal{S}$ which is the unital line $(m)^{\rho}$, where $\rho$ is the unitary polarity defining $\mathcal{U}$, is not incident on any of these circles since the lines joining the absolute points on $(m)^{\rho}$ to $(m)$ are the absolute lines on $(m)$. It follows that $(m)^{\rho}$ together with these $q-1$ circles partition the points of $\mathcal{S}$. We study the images of these circles in $\mathscr{I}^{X}$ under the isomorphism $H$.

For any $m \in \mathcal{K}$, let $\mathcal{M}_{(m)}$ be the set of equivalence classes of non-absolute lines through $(m)$.

Lemma 6.4. For any point $Z$ of the residual $\mathscr{I}^{X}$, the flock $\mathcal{F}(X, Z)$ is given by $H\left(\mathcal{M}_{Z^{H^{-1}}}\right)$. In particular, $H\left(\mathcal{M}_{(m)}\right)$ is the flock $\mathcal{F}\left(X,(m)^{\rho H}\right)$.

Proof. Since $\mathscr{I}$ is classical and $H\left(\mathcal{M}_{(m)}\right)$ is a flock of $\mathscr{I}$ with carriers $X$ and $(m)^{\rho H}, H\left(\mathcal{M}_{(m)}\right)$ is the flock $\mathcal{F}\left(X,(m)^{\rho H}\right)$ by Theorem 5.2. Since there are $q^{2}$ points $Z$ in $\mathscr{I}^{X}$ and $q^{2}$ points $(m)$ on [ $\left.\infty\right]$, the result follows.

\section{Isomorphisms between Dickson-Ganley unitals}

As noted in Sect. 2, two semifields define isomorphic projective planes if and only if the semifields are isotopic; and in the case of a Dickson semifield, $\Pi\left(\mathcal{K}\left(\sigma_{1}\right)\right)$ is isomorphic to $\Pi\left(\mathcal{K}\left(\sigma_{2}\right)\right)$ if and only if $\sigma_{2}=\sigma_{1}$ or $\sigma_{2}=\sigma_{1}{ }^{-1}$. Now consider a Dickson unital $\mathcal{U}(\sigma)$ defined by the polarity $\rho$ of $\Pi(\mathcal{K}(\sigma))$. We provided two proofs, in Sects. 3 and 4 respectively, that $\mathcal{U}(\sigma)$ is not classical. 
Both proofs are intrinsic in nature, that is, they concern incidence patterns in $\mathcal{U}(\sigma)$. But how does one distinguish among these non-classical unitals? In this section, we shall answer this question by constructing from an isomorphism between two Dickson unitals an isotopism between their corresponding Dickson semifields. In particular this also provides a third and extrinsic proof that $\mathcal{U}(\sigma)$ is not classical. Furthermore, we prove that the isotopism gives rise to an isomorphism between the ambient planes, resulting in our main extension theorem. As a consequence we conclude that the automorphism group of $\mathcal{U}(\sigma)$ equals the collineation subgroup of the ambient plane stabilizing the unital.

To begin with, we extend our notations to include the case $\sigma=i d$. Note that $\mathcal{K}(i d)=\mathbb{F}_{q^{2}}, \Pi(\mathcal{K}(i d))=P G\left(2, q^{2}\right)$, and $\mathcal{U}(i d)$ is the classical unital. Moreover, Lemma 4.1 and Corollary 4.2, and all results in Sect. 6 still hold with this extension. For $i=1,2$, consider the unital $\mathcal{U}\left(\sigma_{i}\right)$ embedded in $\Pi\left(\mathcal{K}\left(\sigma_{i}\right)\right)$ defined by the polarity $\rho_{i}$ associated with the involutory automorphisms $\alpha_{i}$ of $\mathcal{K}\left(\sigma_{i}\right)$. For simplicity in notations write $\mathcal{U}_{i}=\mathcal{U}\left(\sigma_{i}\right)$ and $\mathcal{K}_{i}=\mathcal{K}\left(\sigma_{i}\right)$. Let $\operatorname{Isom}\left(\mathcal{U}_{1}, \mathcal{U}_{2}\right)$ be the set of isomorphisms from $\mathcal{U}_{1}$ to $\mathcal{U}_{2}$, and $\operatorname{Isom}^{*}\left(\mathcal{U}_{1}, \mathcal{U}_{2}\right)$ the subset of those which extend to isomorphisms from $\Pi\left(\mathcal{K}_{1}\right)$ to $\Pi\left(\mathcal{K}_{2}\right)$. Our aim is to show that

$$
\operatorname{Isom}\left(\mathcal{U}_{1}, \mathcal{U}_{2}\right)=\operatorname{Isom}^{*}\left(\mathcal{U}_{1}, \mathcal{U}_{2}\right)
$$

Let $\Phi \in \operatorname{Isom}\left(\mathcal{U}_{1}, \mathcal{U}_{2}\right)$. Note that if either $\sigma_{1}$ or $\sigma_{2}$ is the identity, then the corresponding ambient plane has a collineation group acting doubly transitively on the unital. Thus, we may assume $(\infty)^{\Phi}=(\infty)$ and $(0,0)^{\Phi}=(0,0)$. In case both $\sigma_{i} \neq i d$, then by Corollary 3.2 or $4.4, \Phi$ must map $(\infty)$ to $(\infty)$. Moreover, since $\operatorname{Col}\left(\mathcal{U}_{i}\right)$ acts transitively on the affine unital points, we may also assume that $(0,0)^{\Phi}=(0,0)$. Thus, the extension problem is equivalent to showing that

$$
\operatorname{Isom}\left(\mathcal{U}_{1}, \mathcal{U}_{2}\right)_{(\infty),(0,0)}=\operatorname{Isom}^{*}\left(\mathcal{U}_{1}, \mathcal{U}_{2}\right)_{(\infty),(0,0)},
$$

where $\operatorname{Isom}\left(\mathcal{U}_{1}, \mathcal{U}_{2}\right)_{(\infty),(0,0)}$ denotes the subset of isomorphisms mapping $(\infty)$ to $(\infty)$ and $(0,0)$ to $(0,0)$, and similarly for $\operatorname{Isom}^{*}\left(\mathcal{U}_{1}, \mathcal{U}_{2}\right)_{(\infty),(0,0)}$.

Construct $\mathcal{S}_{1}, \mathcal{S}_{2}$ from $\mathcal{U}_{1}, \mathcal{U}_{2}$ as in Sect. 5. Let $\operatorname{Isom}\left(\mathcal{S}_{1}, \mathcal{S}_{2}\right)_{[0]}$ be the set of isomorphisms between $\mathcal{S}_{1}$ and $\mathcal{S}_{2}$ mapping [0] to [0]. We study the relation between $\operatorname{Isom}\left(\mathcal{U}_{1}, \mathcal{U}_{2}\right)_{(\infty),(0,0)}$ and $\operatorname{Isom}\left(\mathcal{S}_{1}, \mathcal{S}_{2}\right)_{[0]}$. Let $\Phi$ be an element of Isom $\left(\mathcal{U}_{1}, \mathcal{U}_{2}\right)_{(\infty),(0,0)}$. Then $\Phi$ maps bijectively the unital lines through $(\infty)$ in $\mathcal{U}_{1}$, which are the points of $\mathcal{S}_{1}$, to the unital lines through $(\infty)$ in $\mathcal{U}_{2}$, which are the points of $\mathcal{S}_{2}$. In particular, [0] $=(\infty) \cdot(0,0)$ is mapped to [0]. Next consider a circle $\langle[m, k]\rangle$ and the set $\mathcal{N}$ of points of $\mathcal{S}_{1}$ on it. Then for each $l \in\langle[m, k]\rangle$, $l^{\Phi}$ intersects each line of $\mathcal{N}^{\Phi}$. By Lemma $4.1, l^{\Phi} \in\left\langle[m, k]^{\Phi}\right\rangle$. In other words, $\mathcal{N}^{\Phi}$ is the set of points of $\mathcal{S}_{2}$ on the circle $\left\langle[m, k]^{\Phi}\right\rangle$. We summarize these observations in the following lemma:

Lemma 7.1. For any $\Phi \in \operatorname{Isom}\left(\mathcal{U}_{1}, \mathcal{U}_{2}\right)_{(\infty),(0,0)}$, let $\widehat{\Phi}$ be the bijection between the points of $\mathcal{S}_{1}$ and $\mathcal{S}_{2}$ given by $[z]^{\widehat{\Phi}}=[z]^{\Phi}$. Then $\langle[m, k]\rangle^{\widehat{\Phi}}=\left\langle[m, k]^{\Phi}\right\rangle$, and $\widehat{\Phi} \in \operatorname{Isom}\left(\mathcal{S}_{1}, \mathcal{S}_{2}\right)_{[0]}$. 
Let $\Lambda: \operatorname{Isom}\left(\mathcal{U}_{1}, \mathcal{U}_{2}\right)_{(\infty),(0,0)} \rightarrow \operatorname{Isom}\left(\mathcal{S}_{1}, \mathcal{S}_{2}\right)_{[0]}$ be the map sending $\Phi$ to $\widehat{\Phi}$.

Lemma 7.2. $\Lambda$ is injective.

Proof. Suppose $\widehat{\Phi}_{1}=\widehat{\Phi}_{2}$, we show that $\Phi_{1}=\Phi_{2}$. Consider a unital line $[m, 0]$ in the circle $\langle[m, 0]\rangle$, noting that $m \neq 0$. Since $\widehat{\Phi}_{1}=\widehat{\Phi}_{2},[m, 0]^{\Phi_{1}} \in\langle[m, 0]\rangle^{\widehat{\Phi}_{1}}=$ $\langle[m, 0]\rangle^{\widehat{\Phi}_{2}} \ni[m, 0]^{\Phi_{2}}$. So $[m, 0]^{\Phi_{1}}$ and $[m, 0]^{\Phi_{2}}$ both define the same circle $\left\langle[m, 0]^{\Phi_{1}}\right\rangle=\left\langle[m, 0]^{\Phi_{2}}\right\rangle$. However, since $(0,0)$ is preserved by both $\Phi_{1}$ and $\Phi_{2}$, we have $[m, 0]^{\Phi_{1}}=[m, 0]^{\Phi_{2}}$ by Corollary 4.2. As for the unital lines on $(\infty)$, $[z]^{\Phi_{1}}=[z]^{\widehat{\Phi}_{1}}=[z]^{\widehat{\Phi}_{2}}=[z]^{\Phi_{2}}$ for any $z$. Since any absolute point off [0] can be written as $(z,-m z)=[m, 0] \cap[z]$, both $\Phi_{1}$ and $\Phi_{2}$ agree on all such points. By considering any two absolute points on a non-absolute line, we see that every non-absolute line has the same image under $\Phi_{1}$ and $\Phi_{2}$. It follows that any absolute point on [0] also has the same image under $\Phi_{1}$ and $\Phi_{2}$. Hence $\Phi_{1}=\Phi_{2}$.

We now make use of the classical inversive plane $\mathscr{I}$ of Sect. 5 to obtain a key property of the maps in $\operatorname{Isom}\left(\mathcal{U}_{1}, \mathcal{U}_{2}\right)_{(\infty),(0,0)}$. First we note that the group isomorphism $h: \operatorname{Aut}(\mathcal{S}) \rightarrow \operatorname{Aut}\left(\mathscr{I}^{X}\right)$ induced by $H: \mathcal{S} \rightarrow \mathscr{I}^{X}$ and given by (6.4) is now replaced by a bijection

$$
h: \operatorname{Isom}\left(\mathcal{S}_{1}, \mathcal{S}_{2}\right)_{[0]} \rightarrow \operatorname{Aut}\left(\mathscr{I}^{X}\right)_{Y}
$$

induced by $H_{i}: \mathcal{S}_{i} \rightarrow \mathscr{I}^{X}, i=1,2$, and given by

$$
H_{1} \Psi^{h}=\Psi H_{2}
$$

for any $\Psi \in \operatorname{Isom}\left(\mathcal{S}_{1}, \mathcal{S}_{2}\right)_{[0]}$. Then Lemma 6.4 applies and we obtain the following property:

Lemma 7.3. Let $\Phi \in \operatorname{Isom}\left(\mathcal{U}_{1}, \mathcal{U}_{2}\right)_{(\infty),(0,0)}$. For any $m \in \mathcal{K}_{1}, \Phi$ maps lines through $(m)$ to lines through $(m)^{\rho_{1} \Phi \rho_{2}}$.

Proof. By Lemmas 7.1 and 7.2, the action of $\Phi$ is determined by $\widehat{\Phi}^{h}$. By Lemma $6.4, H_{1}\left(\mathcal{M}_{(m)}\right)=\mathcal{F}\left(X,(m)^{\rho_{1} H_{1}}\right)$. By Theorem 5.2, this flock is mapped by $\widehat{\Phi}^{h}$ to $\mathcal{F}\left(X,(m)^{\rho_{1} H_{1} \widehat{\Phi}^{h}}\right)$. Again by Lemma 6.4 , this latter flock is given by $H\left(\mathcal{M}_{(m)^{\rho_{1} \Phi \rho_{2}}}\right)$.

The plan is now clear. We shall prove that

$$
\Lambda\left(\operatorname{Isom}\left(\mathcal{U}_{1}, \mathcal{U}_{2}\right)_{(\infty),(0,0)}\right)=\Lambda\left(\operatorname{Isom}^{*}\left(\mathcal{U}_{1}, \mathcal{U}_{2}\right)_{(\infty),(0,0)}\right)
$$

Since $\Lambda$ is injective, this gives (7.1). Thus let $\Phi \in \operatorname{Isom}\left(\mathcal{U}_{1}, \mathcal{U}_{2}\right)_{(\infty),(0,0)}$. We wish to find $\gamma \in \operatorname{Isom}^{*}\left(\mathcal{U}_{1}, \mathcal{U}_{2}\right)_{(\infty),(0,0)}{ }^{1}$ such that $\Lambda(\Phi)=\Lambda(\gamma)$. Since

${ }^{1}$ By Lemma 2 of [13], Isom* $\left(\mathcal{U}_{1}, \mathcal{U}_{2}\right)_{(\infty),(0,0)}=\{\gamma(A, B, C) \mid(A, B, C)$ is an isotopism from $\mathcal{K}_{1}$ to $\left.\mathcal{K}_{2}, A \alpha_{2}=\alpha_{1} A, C \alpha_{2}=\alpha_{1} B\right\}$ where

$$
\gamma(A, B, C):\left\{\begin{array}{ll}
(z, w) \mapsto\left(z^{C}, w^{A}\right) & ,[m, k] \mapsto\left[m^{B}, k^{A}\right] \\
(m) \mapsto\left(m^{B}\right) & ,[z] \mapsto\left[z^{C}\right] \\
(\infty) \mapsto(\infty) & ,[\infty] \mapsto[\infty]
\end{array},\right.
$$

and that $(A, B, C)$ is an isotopism means that $A, B, C$ are additive bijections from $\mathcal{K}_{1}$ to $\mathcal{K}_{2}$ such that $(m z)^{A}=m^{B} z^{C}$ for any $m, z \in \mathcal{K}_{1}$. 
$h: \operatorname{Isom}\left(\mathcal{S}_{1}, \mathcal{S}_{2}\right)_{(\infty),(0,0)} \rightarrow \operatorname{Aut}\left(\mathscr{I}^{X}\right)_{Y}$ defined by $(7.2)$ is a bijection, this is equivalent to the requirement that

$$
h(\Lambda(\Phi))=h(\Lambda(\gamma)) .
$$

By Theorem 6.3, $h(\Lambda(\Phi))$ is of the form $\varphi_{a, 0,0,1, \epsilon}$ given by (6.3). We shall show that $\varphi_{a, 0,0,1, \epsilon}$ determines three additive bijections $A, B$ and $C$ from $\mathcal{K}_{1}$ to $\mathcal{K}_{2}$, constituting an isotopism $(A, B, C)$, from which we can construct a map $\gamma=\gamma(A, B, C)$ satisfying our requirement.

The maps $A, B$ and $C$ are defined as follows.

Let $\Phi \in \operatorname{Isom}\left(\mathcal{U}_{1}, \mathcal{U}_{2}\right)_{(\infty),(0,0)}$. Then for some $a \in \mathbb{F}_{q^{2}}$ and $\epsilon \in \operatorname{Aut}\left(\mathbb{F}_{q^{2}}\right)$,

$$
h(\Lambda(\Phi))=\widehat{\Phi}^{h}=\varphi_{a, 0,0,1, \epsilon} .
$$

We make a change of variable and introduce a parameter $\eta$. Thus write $a=$ $\left(a_{1}+\kappa a_{2}\right)^{\epsilon}$, where $\kappa$ is as in Sect. 5, and let

$$
\phi_{a_{1}, a_{2}, \epsilon}=\varphi_{a, 0,0,1, \epsilon} .
$$

For any $z=x+\lambda y \in \mathcal{K}$, consider the unital line $[x+\lambda y]$ on $(\infty)$. By (6.1), this is mapped to $\left[x+\kappa y^{\sigma}, 1\right]$. Then we have

$$
\begin{aligned}
{\left[x+\kappa y^{\sigma_{1}}, 1\right]^{\phi_{a_{1}, a_{2}, \epsilon}} } & =\left[\left(a_{1}+\kappa a_{2}\right)^{\epsilon}\left(x+\kappa y^{\sigma_{1}}\right)^{\epsilon}, 1\right] \\
& =\left[\left(\left(a_{1} x+\delta a_{2} y^{\sigma_{1}}\right)+\kappa\left(a_{2} x+a_{1} y^{\sigma_{1}}\right)\right)^{\epsilon}, 1\right] \\
& =\left[\left(a_{1} x+\delta a_{2} y^{\sigma_{1}}\right)^{\epsilon}+\kappa^{\epsilon}\left(a_{2} x+a_{1} y^{\sigma_{1}}\right)^{\epsilon}, 1\right] \\
& =\left[\left(a_{1} x+\delta a_{2} y^{\sigma_{1}}\right)^{\epsilon}+\kappa \eta\left(a_{2} x+a_{1} y^{\sigma_{1}}\right)^{\epsilon}, 1\right],
\end{aligned}
$$

where $\eta=\kappa^{\epsilon} \kappa^{-1}$ satisfies $\eta^{2}=\delta^{\epsilon} \delta^{-1}$. Since $\kappa^{q-1}=-1, \eta^{q-1}=1$ and so $\eta \in \mathbb{F}_{q}$.

By Lemma 7.1, (7.2), (6.1), (7.5), (7.6) and (7.7), we have

$$
\begin{aligned}
{[x+\lambda y]^{\Phi} } & =[x+\lambda y]^{\Phi} \\
& =[x+\lambda y]^{H_{1} \widehat{\Phi}^{h} H_{2}^{-1}} \\
& =\left[x+\kappa y^{\sigma_{1}}, 1\right]^{\Phi^{h}} H_{2}^{-1} \\
& =\left[\left(a_{1} x+\delta a_{2} y^{\sigma_{1}}\right)^{\epsilon}+\kappa \eta\left(a_{2} x+a_{1} y^{\sigma_{1}}\right)^{\epsilon}, 1\right]^{H^{-1}} \\
& =\left[\left(a_{1} x+\delta a_{2} y^{\sigma_{1}}\right)^{\epsilon}+\lambda \eta^{\sigma_{2}^{-1}}\left(a_{2} x+a_{1} y^{\sigma_{1}}\right)^{\epsilon \sigma_{2}^{-1}}\right] .
\end{aligned}
$$

Define an additive bijection $C: \mathcal{K}_{1} \longrightarrow \mathcal{K}_{2}$ by (7.8), i.e.

$$
C: x+\lambda y \longmapsto\left(a_{1} x+\delta a_{2} y^{\sigma_{1}}\right)^{\epsilon}+\lambda \eta^{\sigma_{2}^{-1}}\left(a_{2} x+a_{1} y^{\sigma_{1}}\right)^{\epsilon \sigma_{2}^{-1}} .
$$

Next we consider any affine unital line $[m, k]$. Write $m=u+\lambda v$. By Lemma 7.3, this line, which is on $(u+\lambda v)$, is mapped by $\Phi$ to a unital line on $(u+\lambda v)^{\rho_{1} \Phi \rho_{2}}$. By the definition of $\rho_{i}$ and (7.8), we have

$$
\begin{aligned}
(u+\lambda v)^{\rho_{1} \Phi \rho_{2}} & =[u-\lambda v]^{\Phi \rho_{2}} \\
& =\left[\left(a_{1} u-\delta a_{2} v^{\sigma_{1}}\right)^{\epsilon}+\lambda \eta^{\sigma_{2}^{-1}}\left(a_{2} u-a_{1} v^{\sigma_{1}}\right)^{\epsilon \sigma_{2}^{-1}}\right]^{\rho_{2}} \\
& =\left(\left(a_{1} u-\delta a_{2} v^{\sigma_{1}}\right)^{\epsilon}-\lambda \eta^{\sigma_{2}^{-1}}\left(a_{2} u-a_{1} v^{\sigma_{1}}\right)^{\epsilon \sigma_{2}^{-1}}\right) .
\end{aligned}
$$


Define an additive bijection $B: \mathcal{K}_{1} \longrightarrow \mathcal{K}_{2}$ by $(7.10)$, i.e.

$$
B: u+\lambda v \longmapsto\left(a_{1} u-\delta a_{2} v^{\sigma_{1}}\right)^{\epsilon}-\lambda \eta^{\sigma_{2}^{-1}}\left(a_{2} u-a_{1} v^{\sigma_{1}}\right)^{\epsilon \sigma_{2}^{-1}} .
$$

Then

$$
\begin{aligned}
m^{B} z^{C}= & \left(x u+\delta y^{\sigma_{1}} v^{\sigma_{1}}\right)^{\epsilon}\left(a_{1}{ }^{2}-\delta a_{2}{ }^{2}\right)^{\epsilon} \\
& +\lambda \eta^{\sigma_{2}^{-1}}\left[a_{1} a_{1}{ }^{\sigma_{2}^{-1}}\left(x v^{\sigma_{1} \sigma_{2}^{-1}}+y^{\sigma_{1} \sigma_{2}^{-1}} u\right)-a_{2} a_{2}{ }^{\sigma_{2}^{-1}} \delta\left(x^{\sigma_{2}^{-1}} v^{\sigma_{1}}+y^{\sigma_{1}} u^{\sigma_{2}^{-1}}\right)\right. \\
& \left.+a_{1} a_{2}{ }^{\sigma_{2}^{-1}}\left(x^{\sigma_{2}^{-1}} u-x u^{\sigma_{2}^{-1}}\right)+\delta a_{1}{ }^{\sigma_{2}^{-1}} a_{2}\left(y^{\sigma_{1}} v-y v^{\sigma_{1}}\right)\right]^{\epsilon} .
\end{aligned}
$$

Suppose there is an additive bijection $A: \mathcal{K}_{1} \longrightarrow \mathcal{K}_{2}$ completing $B, C$ to an isotopism. Then $A$ is uniquely determined by $B$ and $C$. Consider the following definition of $A$ :

$$
A: s+\lambda t \longmapsto s^{\epsilon}\left(a_{1}^{2}-\delta a_{2}^{2}\right)^{\epsilon}+\lambda \eta^{\sigma_{2}^{-1}}\left(t a_{1} a_{1}^{\sigma_{2}^{-1}}-t^{\sigma_{2}^{-1}} \delta a_{2} a_{2}{ }^{-1}\right)^{\epsilon} .
$$

Note that $A$ is additive, and

$$
\begin{aligned}
(m z)^{A}= & \left(x u+\delta y^{\sigma_{1}} v^{\sigma_{1}}\right)^{\epsilon}\left(a_{1}^{2}-\delta a_{2}{ }^{2}\right)^{\epsilon} \\
& +\lambda \eta^{\sigma_{2}^{-1}}\left[a_{1} a_{1}{ }^{\sigma_{2}^{-1}}(x v+y u)-a_{2} a_{2}{ }^{\sigma_{2}^{-1}} \delta\left(x^{\sigma_{2}^{-1}} v^{\sigma_{2}^{-1}}+y^{\sigma_{2}^{-1}} u^{\sigma_{2}^{-1}}\right)\right]^{\epsilon} .
\end{aligned}
$$

We show that this is sufficient to yield our first main result.

Theorem 7.4. If $\mathcal{U}_{1}$ and $\mathcal{U}_{2}$ are isomorphic, then $\mathcal{K}_{1}$ and $\mathcal{K}_{2}$ are isotopic.

Proof. Suppose $\mathcal{U}_{1}$ and $\mathcal{U}_{2}$ are isomorphic via an isomorphism $\Phi$. We may assume without loss of generality that $\Phi \in \operatorname{Isom}\left(\mathcal{U}_{1}, \mathcal{U}_{2}\right)_{(\infty),(0,0)}$ (see the beginning of this section). Then $h(\Lambda(\Phi))=\phi_{a_{1}, a_{2}, \epsilon}$, for some $a_{1}, a_{2} \in \mathbb{F}_{q}$ and $\epsilon \in \operatorname{Aut}\left(\mathbb{F}_{q}\right)$ by $(7.5)$ and $(7.6)$. We shall prove that $(A, B, C)$, defined by (7.13), (7.11) and (7.9), is an isotopism.

First, suppose $\sigma_{1}=\sigma_{2}=i d$. Since $\delta$ is a nonsquare, $A$ is a bijection. By (7.12) and (7.14), for any $m, z \in \mathcal{K}_{1}$,

$m^{B} z^{C}=(x u+\delta y v)^{\epsilon}\left(a_{1}{ }^{2}-\delta a_{2}{ }^{2}\right)^{\epsilon}+\lambda \eta\left[a_{1}^{2}(x v+y u)-a_{2}^{2} \delta(x v+y u)\right]=(m z)^{A}$.

Hence $(A, B, C)$ is an isotopism.

Second, suppose one of them, which we may assume to be $\sigma_{2}$, is non-identity. We derive necessary conditions on $a_{1}$ and $a_{2}$.

Let $\omega$ be a primitive element of $\mathbb{F}_{q}$. Consider an absolute point $(z,-m z)$ on the affine non-absolute line $\left[m^{\prime},\left(m^{\prime}-m\right) z\right]$ in $\mathcal{U}_{1}$. We have

$$
(z,-m z)^{\Phi}=[z]^{\Phi} \cap\left((0,0)^{\Phi} \cdot(m)^{\rho_{1} \Phi \rho_{2}}\right)=\left[z^{C}\right] \cap\left((0,0) \cdot\left(m^{B}\right)\right)=\left(z^{C},-m^{B} z^{C}\right) .
$$

It follows that

$$
\begin{aligned}
{\left[m^{\prime},\left(m^{\prime}-m\right) z\right]^{\Phi} } & =\left(m^{\prime}\right)^{\rho_{1} \Phi \rho_{2}} \cdot(z,-m z)^{\Phi} \\
& =\left(m^{\prime B}\right) \cdot\left(z^{C},-m^{B} z^{C}\right)=\left[m^{\prime B},\left(m^{\prime}-m\right)^{B} z^{C}\right],
\end{aligned}
$$


where the last equality holds since $B$ is additive. In particular, consider the non-absolute line

$$
l=\left(1,-\frac{1}{2}\right) \cdot\left(\omega,-\frac{\omega}{2} \omega\right)=\left[\frac{\omega+1}{2}, \frac{\omega}{2}\right] .
$$

Since $\left(1,\left(-\frac{1}{2}\right) 1\right) \in\left[\frac{\omega+1}{2},\left(\frac{\omega+1}{2}-\frac{1}{2}\right) 1\right]$ and $\left(\omega,-\frac{\omega}{2} \omega\right) \in\left[\frac{\omega+1}{2},\left(\frac{\omega+1}{2}-\frac{\omega}{2}\right) \omega\right]$, expressing the second coordinate of $l^{\Phi}$ in two ways we obtain

$$
\left(\frac{\omega}{2}\right)^{B} 1^{C}=\left(\frac{1}{2}\right)^{B} \omega^{C} .
$$

By (7.12), this gives $a_{1} a_{2}{ }^{\sigma_{2}^{-1}}\left(\frac{\omega}{2}-\frac{\omega^{\sigma_{2}^{-1}}}{2}\right)=a_{1} a_{2}{ }^{\sigma_{2}^{-1}}\left(\frac{\omega^{\sigma_{2}^{-1}}}{2}-\frac{\omega}{2}\right)$. Since $\omega$ is a primitive element, $\omega^{\sigma_{2}^{-1}} \neq \omega$; it follows that $a_{1} a_{2}=0$. Since exactly one of $a_{1}, a_{2}$ equals 0 and $\delta$ is a nonsquare, $A$ is a bijection.

Next, we consider the non-absolute line

$$
\left(\lambda, \frac{\lambda}{2} \lambda\right) \cdot\left(\lambda \omega,\left(\omega^{-1}-1+\frac{\lambda \omega}{2}\right) \lambda \omega\right)=\left[1-\lambda \frac{1+\omega}{2},-\frac{\delta \omega^{\sigma_{1}}}{2}+\lambda\right] .
$$

Since $\left(\lambda, \frac{\lambda}{2} \lambda\right) \in\left[1-\lambda \frac{1+\omega}{2},\left(1-\frac{\lambda \omega}{2}\right) \lambda\right]$ and $\left(\lambda \omega,\left(\omega^{-1}-1+\frac{\lambda \omega}{2}\right) \lambda \omega\right) \in[1-$ $\left.\lambda \frac{1+\omega}{2},\left(\omega^{-1}-\frac{\lambda}{2}\right) \lambda \omega\right]$, by the same method as above we obtain

$$
\left(1-\frac{\lambda \omega}{2}\right)^{B} \lambda^{C}=\left(\frac{1}{\omega}-\frac{\lambda}{2}\right)^{B}(\lambda \omega)^{C} .
$$

By (7.12) and the fact that $a_{1} a_{2}=0, a_{1} a_{1}^{\sigma_{1}^{-1}}\left(1-\frac{\omega^{\sigma_{1} \sigma_{2}^{-1}}}{\omega}\right)-a_{2} a_{2}^{\sigma_{2}^{-1}} \delta\left(1-\frac{\omega^{\sigma_{1}}}{\omega^{\sigma_{2}^{-1}}}\right)=$ 0 . Thus, if $a_{1}=0$, then $\omega^{\sigma_{1}}=\omega^{\sigma_{2}^{-1}}$. Since $\omega$ is a primitive element, $\sigma_{1}=\sigma_{2}^{-1}$, i.e. $\sigma_{1} \sigma_{2}=i d$. If $a_{2}=0$, then $\omega^{\sigma_{1} \sigma_{2}^{-1}}=\omega$. Hence, $\sigma_{1}=\sigma_{2}$. In either case, it follows that $\sigma_{1}$ is non-identity. By (7.12) and (7.14), we have for any $m, z$ in $\mathcal{K}_{1}$, in the former case,

$m^{B} z^{C}=\left(x u+\delta y^{\sigma_{1}} v^{\sigma_{1}}\right)^{\epsilon}\left(-\delta a_{2}{ }^{2}\right)^{\epsilon}-\lambda \eta^{\sigma_{1}}\left[a_{2} a_{2}{ }^{\sigma_{1}} \delta\left(x^{\sigma_{1}} v^{\sigma_{1}}+y^{\sigma_{1}} u^{\sigma_{1}}\right)\right]^{\epsilon}=(m z)^{A} ;$

in the latter case,

$$
m^{B} z^{C}=\left(x u+\delta y^{\sigma_{1}} v^{\sigma_{1}}\right)^{\epsilon}\left(a_{1}^{2}\right)^{\epsilon}+\lambda \eta^{\sigma_{1}^{-1}}\left[a_{1}{a_{1}}^{\sigma_{1}^{-1}}(x v+y u)\right]^{\epsilon}=(m z)^{A} .
$$

Hence, $(A, B, C)$ is an isotopism.

As a consequence this answers our motivating question.

Corollary 7.5. $\mathcal{U}\left(\sigma_{1}\right)$ is isomorphic to $\mathcal{U}\left(\sigma_{2}\right)$ if and only if $\sigma_{2}=\sigma_{1}$ or $\sigma_{2}=$ $\sigma_{1}^{-1}$.

Proof. Suppose $\mathcal{U}\left(\sigma_{1}\right) \cong \mathcal{U}\left(\sigma_{2}\right)$. By the theorem, $\mathcal{K}\left(\sigma_{1}\right)$ and $\mathcal{K}\left(\sigma_{2}\right)$ are isotopic. By Sandler [26] this implies that $\sigma_{2}=\sigma_{1}$ or $\sigma_{2}=\sigma_{1}{ }^{-1}$. Conversely, it is straightforward to check that $\gamma(A, B, C)$, where 


$$
\begin{aligned}
& A: x+\lambda y \mapsto-\left(\frac{x}{\delta}\right)^{\sigma^{-1}}-\lambda \frac{y}{\sqrt{\delta \delta^{\sigma}}}, \\
& B: x+\lambda y \mapsto-y-\lambda \frac{x}{\sqrt{\delta \delta^{\sigma}}}, \\
& C: x+\lambda y \mapsto y+\lambda \frac{x}{\sqrt{\delta \delta^{\sigma}}},
\end{aligned}
$$

is an isomorphism from $\Pi(\mathcal{K}(\sigma))$ to $\Pi\left(\mathcal{K}\left(\sigma^{-1}\right)\right)$ mapping $\mathcal{U}(\sigma)$ to $\mathcal{U}\left(\sigma^{-1}\right)$.

Furthermore, since $\mathcal{U}(i d)$ is a classical unital, this gives a third and extrinsic proof that a Dickson-Ganley unital is non-classical.

Corollary 7.6. Every Dickson-Ganley unital is non-classical.

We return to the extension problem.

Lemma 7.7. For any $\Phi \in \operatorname{Isom}\left(\mathcal{U}_{1}, \mathcal{U}_{2}\right)_{(\infty),(0,0)}$, there exists an element $\gamma$ in Isom $^{*}\left(\mathcal{U}_{1}, \mathcal{U}_{2}\right)_{(\infty),(0,0)}$ such that $h(\Lambda(\gamma))=h(\Lambda(\Phi))$.

Proof. Let $\Phi \in \operatorname{Isom}\left(\mathcal{U}_{1}, \mathcal{U}_{2}\right)_{(\infty),(0,0)}$. Let $A, B$ and $C$ be the maps determined by $h(\Lambda(\Phi))=\phi_{a_{1}, a_{2}, \epsilon}$ as given by (7.13), (7.11) and (7.9). By Theorem 7.4, $(A, B, C)$ is an isotopism from $\mathcal{K}_{1}$ to $\mathcal{K}_{2}$. It is readily verified that $A \alpha_{2}=\alpha_{1} A$ and $C \alpha_{2}=\alpha_{1} B$. By Lemma 2 of [13], $\gamma=\gamma(A, B, C)$ is in $\operatorname{Isom}^{*}\left(\mathcal{U}_{1}, \mathcal{U}_{2}\right)_{(\infty),(0,0)}$. By (7.10) and $(7.8), h(\Lambda(\gamma))=h(\Lambda(\Phi))$.

We are now ready to prove the main extension theorem.

Theorem 7.8. Every isomorphism from $\mathcal{U}_{1}$ to $\mathcal{U}_{2}$ extends to an isomorphism from $\Pi\left(\mathcal{K}_{1}\right)$ to $\Pi\left(\mathcal{K}_{2}\right)$.

Proof. We prove (7.1). By Lemma 7.7, we have (7.4). This is equivalent to (7.3). But (7.3) implies (7.1).

By taking $\sigma_{1}=\sigma_{2}$, we conclude below that the automorphism group of a Dickson-Ganley unital is the collineation subgroup of the ambient plane stabilizing the unital. Note that this contains the classical result of O'Nan [22].

Corollary 7.9. $\operatorname{Aut}(\mathcal{U})=\operatorname{Col}(\mathcal{U})$

Remark 7.10. The Dickson-Ganley unital $\mathcal{U}$ is defined by the unitary polarity $\rho$ associated to the involutory automorphism $\alpha$. By Ganley [13], a unitary polarity of $\Pi(\mathcal{K})$ is determined by an autotopism $(A, B, C)$ of $\mathcal{K}$ with $A^{2}=$ $i d$ and $B C=i d$. Using results of Albert, Sandler [26] noted that for any autotopism $(A, B, C)$ of $\mathcal{K}, B$ is given by $(x+\lambda y)^{B}=a x^{\epsilon}+b y^{\epsilon}+\lambda\left(c x^{\epsilon}+d y^{\epsilon}\right)$ for $a, b, c, d \in \mathbb{F}_{q}, a d-b c \neq 0$, and $\epsilon \in \mathbb{F}_{q}$. In the case of $\rho, A=B=C=\alpha$. Computations (with details given in [18]) show that every unitary polarity of $\Pi(\mathcal{K})$ is determined by an autotopism of the form $\left(\alpha, a \alpha, a^{-1} \alpha\right)$, for $a \in \mathbb{F}_{q}^{*}$, and is conjugate to $\rho$. There is thus just one class of Dickson-Ganley polar unitals, and the results we have obtained are true for all Dickson-Ganley unitals.

Remark 7.11. The structure of $\operatorname{Col}(\mathcal{U})$ as well as that of the autotopism group of $\mathcal{K}$ are completely determined in [19]. 
Open Access. This article is distributed under the terms of the Creative Commons Attribution License which permits any use, distribution, and reproduction in any medium, provided the original author(s) and the source are credited.

\section{References}

[1] Abatangelo, V., Larato, B.: Polarity and transitive parabolic unitals in translation planes of odd order. J. Geom. 74, 1-6 (2002)

[2] Albert, A.A.: On nonassociative division algebras. Trans. Am. Math. Soc. 72, 296-309 (1952)

[3] Albert, A.A.: Finite division algebras and finite planes. In: Bellman, R., et al. (eds.) Combinatorial Analysis: Proceedings of the 10th Symposium in Appled Mathematics, vol. 10 of Symposia in Appl. Math., pp. 53-70. American Mathematical Society, Providence (1960)

[4] Albert, A.A.: Generalized twisted fields. Pacific J. Math. 11, 1-8 (1961)

[5] Barwick, S.G., O'Keefe, C.M.: Unitals and inversive planes. J. Geom. 58, 43-52 (1961)

[6] Burmester, M.V.D.: On the commutative non-associative division algebras of even order of L.E. Dickson. Rend. Mat. Appl. 21, 143-166 (1962)

[7] Dembowski, P.: Finite Geometries. Springer, Berlin (1968)

[8] Dembowski, P., Hughes, D.R.: On finite inversive planes. J. Lond. Math. Soc. 40, 171-182 (1965)

[9] Dickson, L.E.: On commutative linear algebras in which division is always uniquely possible. Trans. Am. Math. Soc. 7, 514-522 (1906)

[10] Dickson, L.E.: Linear algebras with associativity not assumed. Duke Math. J. 1, 113-125 (1935)

[11] Fisher, J.C., Thas, J.A.: Flocks in PG(3,q). Math. Z. 169, 1-11 (1979)

[12] Ganley, M.J.: Polarities in translation planes. Geom. Dedicata 1, 103-116 (1972)

[13] Ganley, M.J.: A class of unitary block designs. Math. Z. 128, 34-42 (1972)

[14] Hall, M.: Projective planes. Trans. Am. Math. Soc. 54, 229-277 (1943)

[15] Hirschfeld, J.W.P.: Projective Geometries over Finite Fields, 2nd edn. Clarendon, Oxford (1998)

[16] Hoffman, A.J.: Chains in the projective line. Duke Math. J. 18, 827-830 (1951)

[17] Hughes, D.R., Piper, F.C.: Projective Planes. Springer, Heidelberg (1973)

[18] Hui, A.M.W., Law, H.F., Tai, Y.K., Wong, P.P.W.: A note on unitary polarities in finite Dickson semifield planes. J. Geom. (submitted)

[19] Hui, A.M.W., Tai, Y.K., Wong, P.P.W.: The structures of the autotopism group of the commutative Dickson semifield and the stabilizer of the Ganley unital embedded in the corresponding semifield plane. Innov. Incid. Geom. (submitted)

[20] Hui, A.M.W., Wong, P.P.W.: Non-classical polar unitals in finite Figueroa planes. J. Geom. 103, 263-273 (2012)

[21] Knuth, D.E.: Finite semifields and projective planes. J. Algebra 2, 182-217 (1965)

[22] O'Nan, M.E.: Automorphisms of unitary block designs. J. Algebra 20, 495-511 (1965) 
[23] Orr, W.F.: The Miquelian Inversive plane $I P(q)$ and the associated projective planes. Ph.D. Thesis, Univ. Wisconsin, Madison (1973)

[24] Pickert, G.: Projective Ebenen. Springer, Berlin (1955)

[25] Piper, F.C.: Unitary block designs. In: Wilson, R.J. (eds.) Graph Theory and Combinatorics. Research Notes in Mathematics, vol. 34, pp. 98-105. Pitman Advanced Publishing Program, Boston (1979)

[26] Sandler, R.: The collineation groups of some finite projective planes. Portugal. Math. 21, 189-199 (1962)

[27] Thas, J.A.: Flocks of finite egglike inversive planes. In: Barlotti A. (eds.) Finite Geometric Structures and their Applications (C.I.M.E., II Ciclo, Bressanone, 1972), pp. 189-191. Edizioni Cremonese, Rome (1973)

[28] Thas, J.A., Thas, K., Van Maldeghem H.: Translation Generalized Quadrangles. In: Series in Pure Mathematics, vol. 26. World Scientific, Singapore (2006)

[29] Van der Waerden, B.L., Smid, L.J.: Eine Axiomatik der Kreisgeometrie und der Laguerregeometrie. Math. Ann. 110, 753-776 (1935)

[30] Wilbrink, H.: A characterization of the classical unitals. In: Johnson, N.L. et al. (eds.) Finite Geometries. Lecture Notes Pure Appl. Math., vol. 82, pp. 445-454. Dekker, New York (1983)

Alice M. W. Hui, Yee Ka Tai and Philip P. W. Wong

Department of Mathematics

The University of Hong Kong

Hong Kong, China

e-mail: ppwwong@maths.hku.hk

Hiu Fai Law

Mathematisches Seminar

Universität Hamburg

Hamburg, Germany

Received: June 30, 2012.

Revised: July 17, 2013. 